\title{
Excited State Dynamics of 7-Deazaguanosine and Guanosine 5'-Monophosphate in Aqueous Solution
}

\author{
Sarah E. Krul, ${ }^{1, \dagger}$ Sean J. Hoehn, ${ }^{1, \dagger}$ Karl Feierabend, ${ }^{1,2}$ Carlos E. Crespo-Hernández*,1 \\ ${ }^{1}$ Department of Chemistry, Case Western Reserve University, Cleveland, Ohio 44106, USA \\ 2 Permanent address: Department of Chemistry, The College of Wooster, Wooster, Ohio 44691, \\ USA
}

* email of corresponding author: carlos.crespo@case.edu, ${ }^{\dagger}$ these authors contributed equally to this work

\section{ORCID}

Sarah E. Krul: https://orcid.org/0000-0003-4201-039X

Sean J. Hoehn: https://orcid.org/0000-0002-8282-7807

Karl Feierabend: https://orcid.org/0000-0002-4221-2584

Carlos E. Crespo-Hernández: https://orcid.org/0000-0002-3594-0890

\begin{abstract}
Minor structural modifications to the DNA and RNA nucleobases have a significant effect on their excited state dynamics and electronic relaxation pathways. In this study, the excited state dynamics of 7-deazaguanosine and guanosine 5'-monophosphate are investigated in aqueous solution using femtosecond broadband transient absorption spectroscopy following excitation at $267 \mathrm{~nm}$. The transient absorption spectra are collected under experimental conditions that eliminate the requirement to correct the data for the formation of hydrated electrons, resulting from the two-photon ionization of the solvent. The data is fitted satisfactorily using a two-component sequential kinetic model, yielding lifetimes of $210 \pm 50$ fs and $1.80 \pm$ $0.02 \mathrm{ps}$, and $682 \pm 40 \mathrm{fs}$ and $1.4 \pm 0.03 \mathrm{ps}$, for 7-deazaguanosine and guanosine 5'monophosphate, respectively. By analyzing the results from steady-state, time-resolved, and computational calculations, the following relaxation mechanism is proposed for 7deazaguanosine, $\mathrm{S}_{2}\left(\mathrm{~L}_{\mathrm{b}}\right) \rightarrow \mathrm{S}_{1}\left(\mathrm{~L}_{\mathrm{a}}\right) \rightarrow \mathrm{S}_{0}$, whereas a $\mathrm{S}_{2}\left(\mathrm{~L}_{\mathrm{b}}\right) \rightarrow \mathrm{S}_{1}\left(\mathrm{~L}_{\mathrm{a}}\right) \rightarrow \mathrm{S}_{0}($ hot $) \rightarrow \mathrm{S}_{0}$ relaxation mechanism is proposed for guanosine 5'-monophosphate. Interestingly, longer lifetimes for both the $\mathrm{L}_{b} \rightarrow \mathrm{L}_{\mathrm{a}}$ and the $\mathrm{L}_{\mathrm{a}} \rightarrow \mathrm{S}_{0}$ internal conversion pathways are obtained for 7-deazaguanosine compare to guanosine 5'-monophosphate. Collectively, the results demonstrate that substitution of a single nitrogen for a methine $(\mathrm{C}-\mathrm{H})$ group at position seven of the guanine moiety stabilizes the ${ }^{1} \pi \pi^{*} L_{b}$ and $L_{a}$ states and alters the topology of their potential energy surfaces in such a way that the population dynamics of both internal conversion pathways in 7-deazaguanosine are significantly slowed down compared to those in guanosine 5'-monophosphate.
\end{abstract}




\section{Introduction}

Modern time-resolved spectroscopic experiments have shown that the canonical DNA monomers dissipate the excess electronic energy arising from absorption of UV radiation primarily through internal conversion from the ${ }^{1} \pi \pi^{*}$ states to the ground state $\left(\mathrm{S}_{0}\right)$ within hundreds of femtoseconds. ${ }^{1-3}$ This rapid dissipation of excess electronic energy allows nucleobases to be largely photostable and uniquely placed as the building blocks of life on Earth. Vast experimental and computational evidence have been presented demonstrating that minor chemical modifications of the pyrimidine and purine core can alter the electronic relaxation pathways significantly by modifying the topology of their excited state potential energy surfaces. ${ }^{4-15}$ Depending on the specific site of modification on the core, access to specific conical intersections can be made less favorable, often resulting in higher yields of longer-lived excited states and a decrease in photostability.
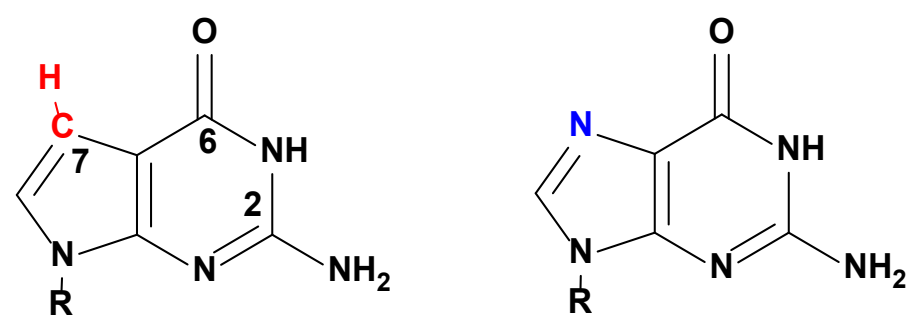

Scheme 1. Structures of 7-deazaguanosine (7dza) and guanosine 5'-monophosphate (GMP). R represents a 2'-deoxyribose group in 7dza and a ribose 5'-monophosphate group in GMP. The common nucleobase ring numbering is shown.

7-Deazaguanosine (7dza) differs in structure from guanosine solely by the replacement of a nitrogen atom with a $\mathrm{C}-\mathrm{H}$ group at the position seven of the guanine moiety (Scheme 1). This minor substitution results in ca. $25 \%$ reduction in the oxidation potential, from $1.24 \mathrm{~V}$ in guanosine to $0.95 \mathrm{~V}$ in $7 \mathrm{dza},{ }^{16-21}$ which has enabled the use of $7 \mathrm{dza}$ as a molecular probe to 
investigate charge transfer dynamics in DNA. ${ }^{20,22,23}$ To the best of our knowledge, however, the electronic relaxation mechanism of $7 \mathrm{dza}$ has not been investigated, precluding a direct comparison with the excited-state dynamics of other purine monomers.

In this contribution, steady-state and time-resolved experiments are presented for $7 \mathrm{dza}$ in aqueous phosphate buffer solution at $\mathrm{pH}$ 6.8. The laser experimental conditions are optimized to eliminate the conventional requirement to correct the transient absorption data for the absorption of hydrated electrons formed from the two-photon ionization of the water solvent when excitation is performed at $267 \mathrm{~nm}$. In addition, newly collected broadband transient absorption experiments for GMP are compared with those collected for $7 \mathrm{dza}$ under equal experimental conditions. GMP is selected in this study for comparison with $7 \mathrm{dza}$ because its excited-state dynamics have been investigated in reasonable details from both experimental and computational perspectives, ${ }^{24-29}$ but also because of its biological relevance. The experimental results are also supplemented with ground and excited-state calculations at the density functional level of theory that take into consideration the dielectric constant of the solvent.

\section{Results}

\section{A. Steady-state absorption spectroscopy}

Figure 1 shows the steady-state absorptivity spectra of 7-deazaguanosine (7dza) and guanosine 5'-monophosphate (GMP) in aqueous phosphate buffer solution at $\mathrm{pH}$ 6.8. At this $\mathrm{pH}$, both molecules are in their keto tautomeric form. ${ }^{30} 7 \mathrm{dza}$ exhibits a band maximum at $259 \mathrm{~nm}$ and a shoulder at ca. $285 \mathrm{~nm}$, whereas GMP exhibits a band maximum at $252 \mathrm{~nm}$ and a shoulder at ca. $275 \mathrm{~nm}$. These results are consistent with those reported in literature, ${ }^{2,24,27}$ for the two lowest lying excited single $\pi \pi^{*}$ transitions, commonly referred to as the $\mathrm{L}_{b}\left(\mathrm{~S}_{2}\right)$ and $\mathrm{L}_{\mathrm{a}}\left(\mathrm{S}_{1}\right)$ states, 
respectively, using Platt's notation. ${ }^{31}$ Relative to GMP, 7 dza absorption band maxima are redshifted by 7 and $10 \mathrm{~nm}$, respectively. Attempts were made to record steady-state fluorescence spectra for these molecules but were unsuccessful due to the relative low sensitivity of the spectrometer available to us.

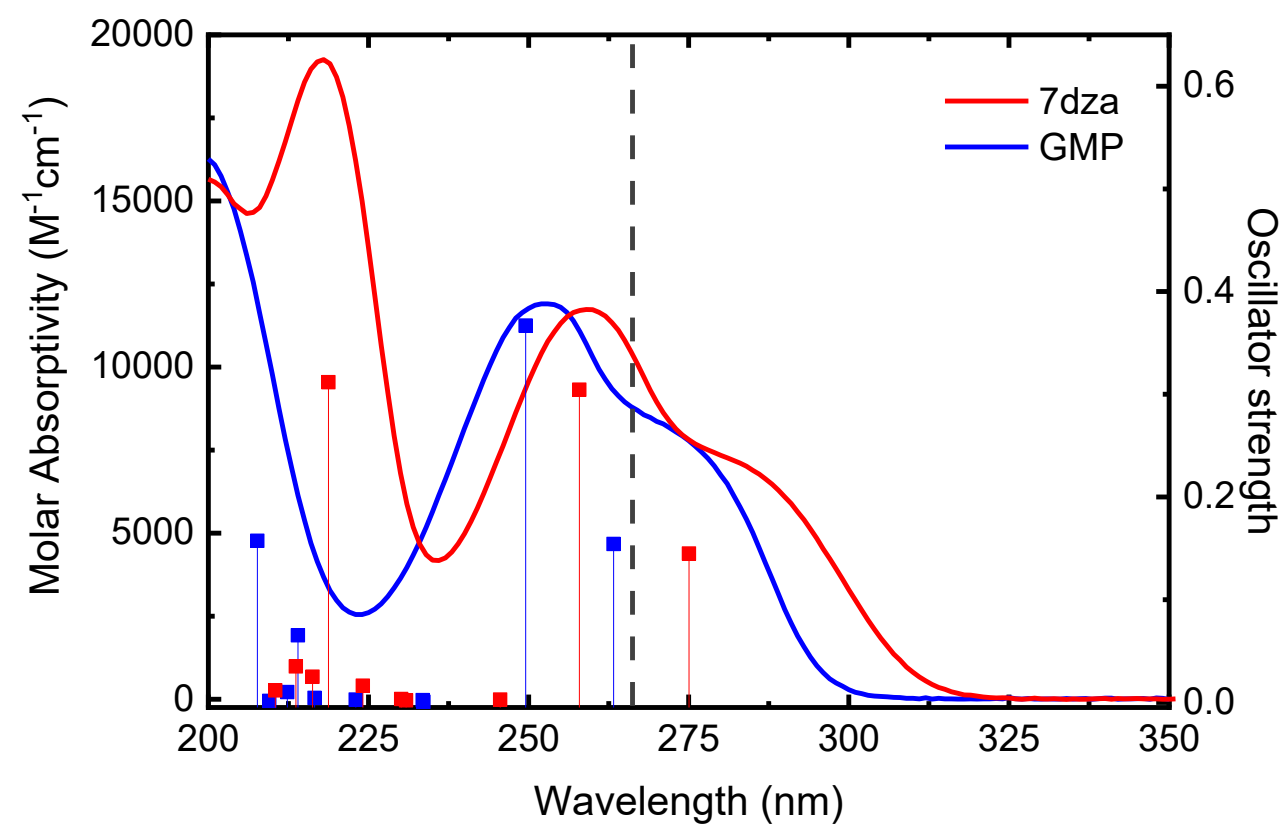

Figure 1. Ground-state absorptivity spectra of 7dza (red) and GMP (blue) in phosphate buffer aqueous solution at $\mathrm{pH}$ 6.8. Vertical lines represent the time-dependent density functional theory (TD-DFT) calculated vertical excitation energies for $7 \mathrm{dza}$ and GMP in the syn-sugar conformations using water as the solvent and the associated oscillator strengths at the TDPBE0/IEFPCM/6-311+G(d,p)//B3LYP/IEFPCM/6-311+G(d,p) level of theory. The dashed line indicates the excitation wavelength of $267 \mathrm{~nm}$ used in the laser experiments.

\section{B. Quantum chemical calculations}

The ground state geometries of both 7dza and GMP were optimized at the B3LYP/IEFPCM/6-311+G(d,p) level of theory in water, followed by calculation of the vertical excitation energies at the TD-PBE0/IEFPCM/6-311+G(d,p) level of theory. Both syn and antisugar conformations were optimized for both molecules. The syn-sugar conformation is the most 
stable in both $7 \mathrm{dza}$ and GMP in water, with the anti-conformation being 1.4 and $3.4 \mathrm{kcal} / \mathrm{mol}$ higher in energy, respectively, at the TD-PBE0/IEFPCM/6-311+G(d,p) level of theory (see Tables S1 and S2). The purine chromophore of both optimized structures is planar, while the amino group at the $\mathrm{C} 2$ is pyrimidalized in both molecules.

The energies and characters of the lowest two singlet and three triplet states for each molecule are listed in Table 1 for the syn-sugar conformations. The percent contributions of the single electron transitions for each excited state are tabulated in Tables S3 and S5 for the synsugar conformations, whereas Tables S5 and S6 collect the energies and characters of the lowest two singlet and three triplet states and percent contributions of the single electron transitions for each excited state for the anti-sugar conformations. The Kohn-Sham orbitals are reported in Figures S1 and S2 syn-sugar conformations and in Figures S3 and S4 for the anti-sugar conformations.

The two lowest energy excited singlet states have $\pi \pi^{*}$ character for both 7dza and GMP. These states are slightly red shifted by approximately $0.2 \mathrm{eV}$ for 7 dza relative to GMP, which corresponds well with the red shift in steady-state absorption spectra shown in Figure 1. The equal ordering and character of electronic states for $7 \mathrm{dza}$ and GMP in water suggests that $\mathrm{N}$-for$\mathrm{CH}$ substitution at the position seven of the purine chromophore does not affect the order of the lowest energy excited singlet states in the Franck-Condon region. It is important to note that both the $L_{a}$ and $L_{b}$ singlet excited states of 7dza and GMP can be simultaneously populated upon excitation at $267 \mathrm{~nm}$ in aqueous solution. 
Table 1. Vertical excitation energies (in $\mathrm{eV}$ ) for the lowest two excited singlet states and three excited triplet states of the syn-sugar conformation of 7-deazaguanosine (7dza) and guanosine 5'monophosphate (GMP) in water calculated at the TD-PBE0/IEFPCM/6$311+\mathrm{G}(\mathrm{d}, \mathrm{p}) / / \mathrm{B} 3 \mathrm{LYP} / \mathrm{IEFPCM} / 6-311+\mathrm{G}(\mathrm{d}, \mathrm{p})$ level of theory. The respective oscillator strengths are listed in parentheses.

\begin{tabular}{lll}
\hline State & 7dza & GMP \\
\hline $\mathrm{S}_{1}\left(\pi \pi^{*}, \mathrm{~L}_{\mathrm{a}}\right)$ & $4.7(0.1448)$ & $4.9(0.1541)$ \\
$\mathrm{S}_{2}\left(\pi \pi^{*}, \mathrm{~L}_{\mathrm{b}}\right)$ & $5.0(0.3044)$ & $5.2(0.3668)$ \\
$\mathrm{T}_{1}\left(\pi \pi^{*}\right)$ & 3.6 & 3.6 \\
$\mathrm{~T}_{2}\left(\pi \pi^{*}\right)$ & 3.6 & 3.9 \\
$\mathrm{~T}_{3}\left(\pi \pi^{*}\right)$ & 4.6 & 4.8
\end{tabular}

\section{Femtosecond broadband transient absorption spectroscopy}

Figure 2 shows the transient absorption spectra for 7dza (a-b) and GMP (c-d) following excitation at $267 \mathrm{~nm}$ in aqueous buffer solution at $\mathrm{pH}$ 6.8. One-photon excitation conditions were used to avoid the two-photon ionization of the water solvent and, therefore, the need for correction of the hydrated electron absorption band (see Methods for details). Excitation of 7dza results in the observation of transient spectra with a band maximum above $330 \mathrm{~nm}$ and a simultaneous band with absorption maximum around $550 \mathrm{~nm}$ that blue shifts to ca. $480 \mathrm{~nm}$ within the cross-correlation of the pump and probe beams (Figure 2a). Subsequently, both absorption bands decay uniformly within ca. $12 \mathrm{ps}$, after which point no further transient absorption signal is detected (Figure 2b). Figure 2c shows the transient absorption spectra of GMP within the cross-correlation of the pump and probe beams. The absorption spectrum at time zero has maxima at ca. 330,425 , and $575 \mathrm{~nm}$. The transient absorption spectra fully decay within ca. 10 ps. However, as shown in Figure $2 d$ and Figure S5b, the band with a maximum around $575 \mathrm{~nm}$ decays faster than the absorption bands at ca. 350 and $475 \mathrm{~nm}$. 

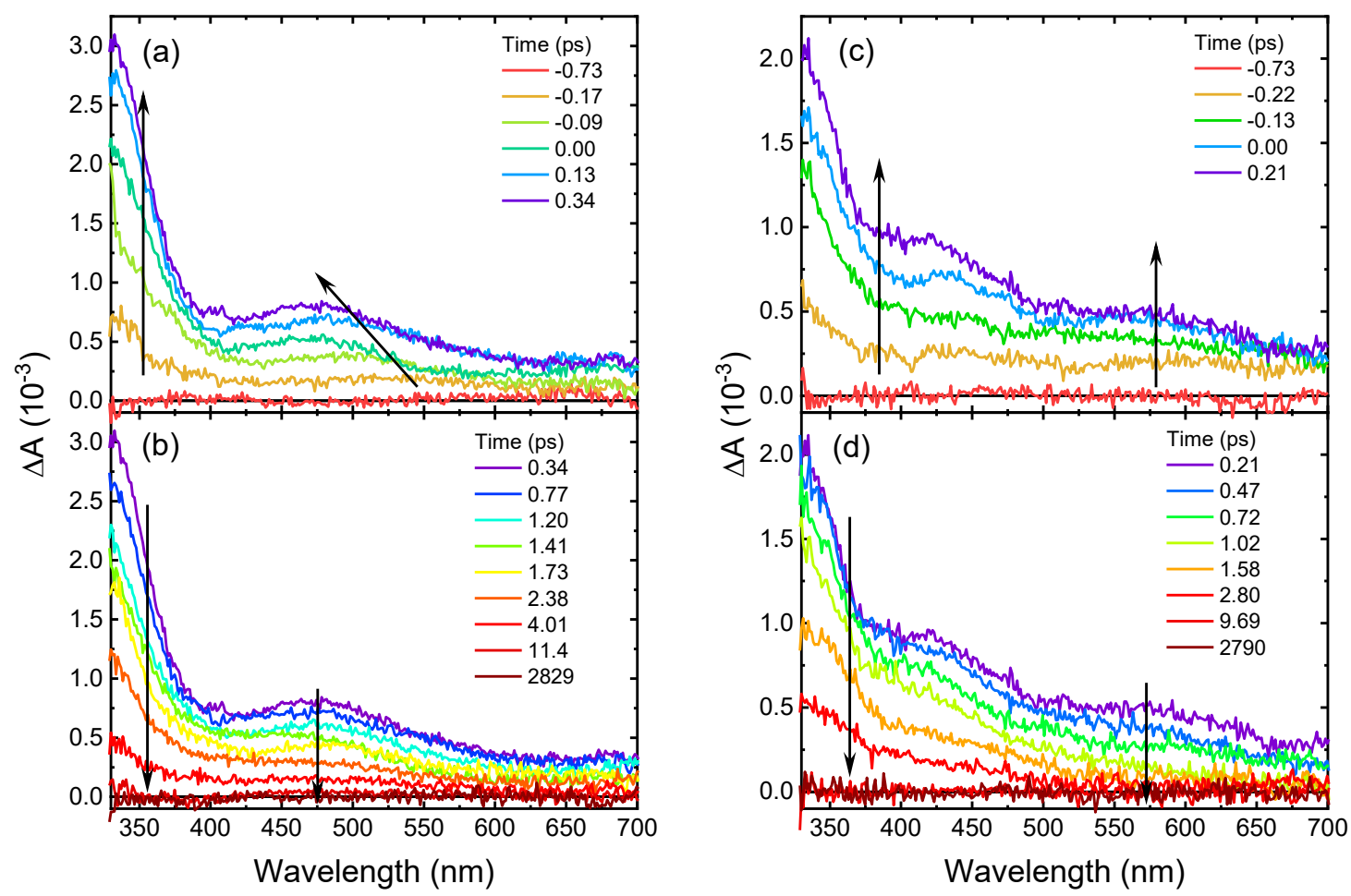

Figure 2. Spectral evolution of the transient absorption spectra of $7 \mathrm{dza}$ (left) and GMP (right) following excitation at $267 \mathrm{~nm}$ in phosphate buffer $\mathrm{pH} 6.8$.

A two-component sequential kinetic model was used to globally fit satisfactorily the transient absorption data for both 7dza and GMP, yielding lifetimes of $210 \pm 50$ fs and $1.80 \pm$ $0.02 \mathrm{ps}$, and of $682 \pm 40 \mathrm{fs}$ and $1.4 \pm 0.03 \mathrm{ps}$, respectively. Figure 3 shows representative kinetic decay traces over the initial 50 ps time window. The solid lines represent the best fit obtained through global and target analysis of the full broadband transient absorption data for each molecule. The evolution associated difference spectra (EADS) extracted from the global and targeted analyses of the broadband data are shown in Figure 4. 


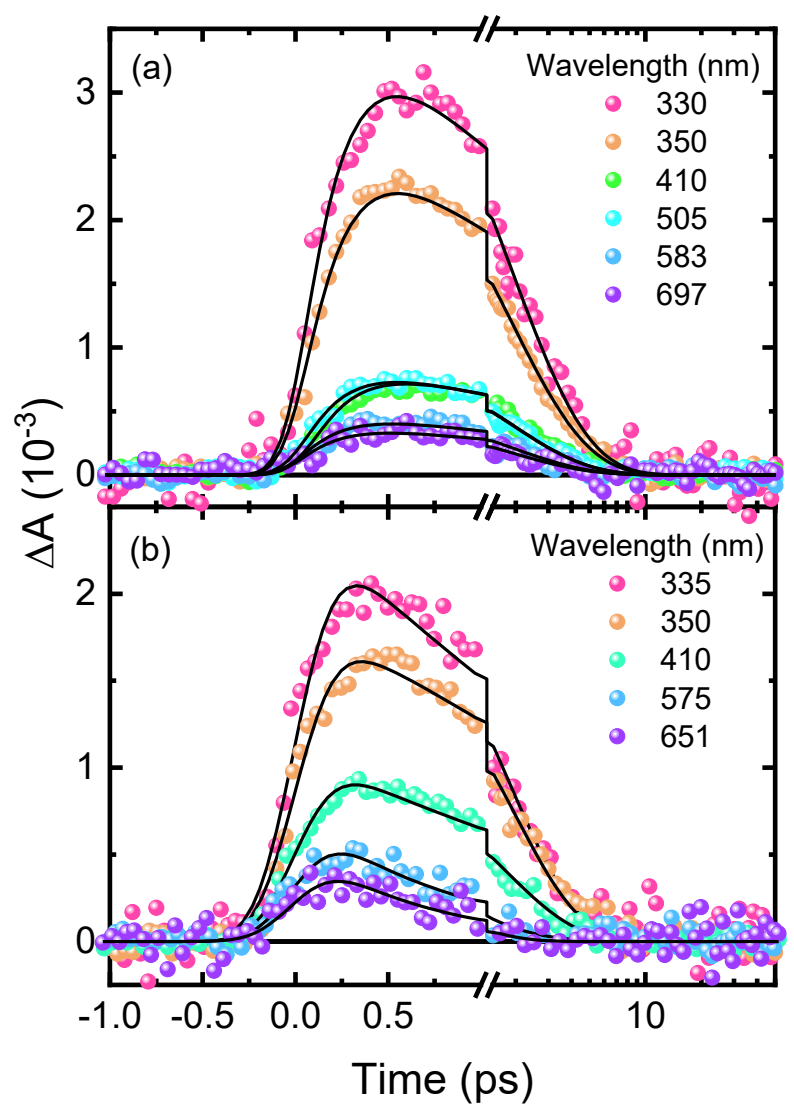

Figure 3. Representative kinetic decay traces of 7dza (a) and GMP (b) at select probe wavelengths globally fit with a two-component sequential kinetic model. 


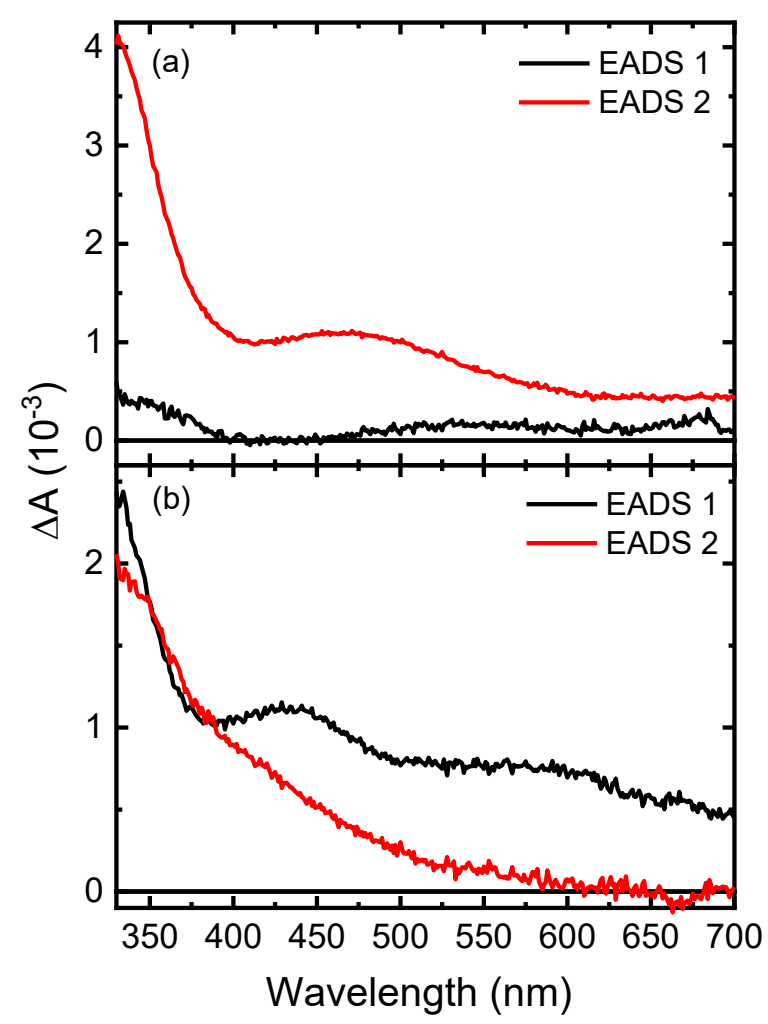

Figure 4. Evolution associated difference spectra of 7dza (a) and GMP (b) globally fit with a two-component sequential kinetic model.

\section{Discussion}

\section{A. Excited-state relaxation mechanism for $7 \mathrm{dza}$ in aqueous solution}

The absorptivity spectrum and the vertical excitation energies and oscillator strengths reported in Figure 1 and Table 1, respectively, suggest that both the $\mathrm{S}_{1}\left(\pi \pi^{*}, \mathrm{~L}_{\mathrm{a}}\right)$ and $\mathrm{S}_{2}\left(\pi \pi^{*}, \mathrm{~L}_{\mathrm{b}}\right)$ states are populated simultaneously following excitation of $7 \mathrm{dza}$ at $267 \mathrm{~nm}$ in aqueous solution. Using a linear combination of two Gaussian functions to model the absorptivity spectrum of $7 \mathrm{dza}$ in the spectral region from ca. 230 to $330 \mathrm{~nm}$ (Figure S6a), it is estimated that 95\% of the initial excited state population reaches the $\mathrm{L}_{b}$ state, whereas only about $5 \%$ reaches the $\mathrm{L}_{\mathrm{a}}$ state. As shown in Figure 2a,b, the excited state population fully decays within about 12 ps and the 
broadband data can be satisfactorily fit with a two-component sequential kinetic model, yielding lifetimes of $210 \mathrm{fs}$ and $1.8 \mathrm{ps}$. Then, we associate the first lifetime to two processes: internal conversion from the $\mathrm{L}_{b}$ state to the $\mathrm{L}_{\mathrm{a}}$ state and direct population of the $\mathrm{L}_{\mathrm{a}}$ state, both occurring within the cross-correlation of the pump and probe beams. This assignment can explain the blue shift in the transient absorption spectra from 550 to $480 \mathrm{~nm}$ observed in Figure 2a. As a result, the black EADS reported in Figure 4a is primarily associated with the excited-state absorption of the $\mathrm{L}_{b}$ state. The second lifetime is then assigned to internal conversion from the $\mathrm{L}_{a}$ state to the ground state. Therefore, the red EADS in Figure $4 \mathrm{a}$ is associated with the excited-state absorption of the $\mathrm{L}_{\mathrm{a}}$ state, which population decays to the ground state in $1.8 \mathrm{ps}$.

\section{B. Excited-state relaxation mechanism for GMP in aqueous solution}

As observed for $7 \mathrm{dza}$, the absorptivity spectrum and the vertical excitation energies presented in Figure 1 and Table 1, respectively, suggest that both the $S_{1}\left(\pi \pi^{*}, L_{a}\right)$ and $S_{2}\left(\pi \pi^{*}, L_{b}\right)$ states are populated simultaneously upon excitation at $267 \mathrm{~nm}$ in water. The absorptivity spectrum of GMP from ca. 230 to $330 \mathrm{~nm}$ was modeled using a linear combination of two Gaussian functions as was done for $7 \mathrm{dza}$ (Figure S6b) to estimate the fraction of the initial excited state population that reaches each state. According to this analysis, $79 \%$ of the initial population reaches the $\mathrm{L}_{\mathrm{b}}$, whereas $21 \%$ reaches the $\mathrm{L}_{\mathrm{a}}$ state. The transient absorption data and the vertical excitation energies suggest that the first lifetime is most likely assigned to internal conversion from the $S_{1}\left(\pi \pi^{*}, L_{a}\right)$ state to the ground state. The population reaching the $L_{b}$ state, internally convert to the $\mathrm{L}_{\mathrm{a}}$ state within the time resolution of our setup and cannot be clearly resolved. In other words, excitation of GMP at $267 \mathrm{~nm}$ populates both the $\mathrm{L}_{b}$ and $\mathrm{L}_{\mathrm{a}}$ states simultaneously. However, the population reaching the $\mathrm{L}_{b}$ state internally convert to the $\mathrm{L}_{\mathrm{a}}$ state in an ultrafast time scale, and the $\mathrm{L}_{\mathrm{a}}$ state population then decays to the ground state with a 680 
fs lifetime. The decay of the excited state population in the $\mathrm{L}_{\mathrm{a}}$ state is followed by a slower decay of a broad transient absorption with a global lifetime of $1.4 \mathrm{ps}$. The second lifetime is assigned to energy transfer of the vibrationally-excited ground state to the solvent molecules (i.e., vibrational cooling dynamics). Therefore, the black EADS in Figure $4 \mathrm{~b}$ primarily represents the excitedstate absorption spectrum of the $\mathrm{L}_{\mathrm{a}}$ state, whereas the red EADS primarily represents the absorption spectrum of the vibrationally-excited ground state.

The transient absorption results reported in this study for GMP generally agree well with earlier work done by Karunakaran and Cheng, ${ }^{24,25}$ who observed similar transient absorption spectra for GMP as those reported in Figure 2c,d. However, there is a slight discrepancy in the relative intensity of the UV and visible transient absorption bands around 425 and $600 \mathrm{~nm}$, respectively. Our results show higher intensity for the absorption bands at smaller probe wavelengths than ca. $500 \mathrm{~nm}$, whereas work by Karunakaran and Cheng show approximately equal intensity for both bands at ca. 450 and $600 \mathrm{~nm}$. This may be associated to the different instrument response function (IRF) of the transient absorption setups, particularly if the IRF of both of their systems is shorter than that of our setup (i.e., of ca. $250 \pm 50 \mathrm{fs}$ ). In such a case, the amplitude of the visible absorption band assigned to excited-state absorption of the $\mathrm{L}_{\mathrm{a}}$ state may be underestimated in our transient absorption experiments because a significant fraction of this excited-state population would have been decayed within the cross-correlation of the pump and probe beams. Another factor that could explain the discrepancy between the relative intensities of the 450 and $600 \mathrm{~nm}$ absorption bands for GMP in Figure 2c,d versus in earlier experiments is an over subtraction of the solvent signal at probe wavelengths smaller than ca. $500 \mathrm{~nm}$ in the latter experiments. Solvent signal subtraction is required when a relatively high photon density at $267 \mathrm{~nm}$ is used for excitation, resulting in the formation of hydrated electrons as a consequence 
of two-photon ionization of the water solvent. ${ }^{32}$ It should be remarked at this point that we have also observed signal from the water solvent in the spectral probe region from 320 to $450 \mathrm{~nm}$ that increases linearly with an increase in the pump photon density at $267 \mathrm{~nm}$ (see Methods section below). In our experience, the combination of the one and two-photon signals originating from the solvent makes the correction of the transient absorption spectra of GMP (or any other molecule excited at $267 \mathrm{~nm}$ in aqueous solution) for these solvent signals a challenge because at probe wavelengths around $450 \mathrm{~nm}$ both one- and two-photon solvent signals overlap. This is the reason for which we optimized the experimental conditions to eliminate the need to correct the transient absorption data of GMP and 7dza for the one and two-photon solvent signals.

We note that recent computational studies for guanine monomers at the CASSCF and DFT levels of theory have suggested that upon moving away from the FC region, the $\mathrm{L}_{b}$ state quickly reaches a conical intersection with the lower lying $\mathrm{L}_{\mathrm{a}}$ state. ${ }^{24,28,29}$ This relaxation pathway is thought to occur in less than $50 \mathrm{fs}$. Hence, it is likely that such ultrafast internal conversion process cannot be resolved with our experimental setup, as proposed above. We note that previous experimental investigations using fs-transient absorption and fs-fluorescence spectroscopy have reported three lifetimes for GMP compared to the two lifetimes reported in this study. ${ }^{24-26}$ Obviously, our broadband data can equally be fitted with three lifetimes of similar magnitude as those used in those previous works. However, the use of a three-component sequential model does not improve the quality of the fits significantly enough to justify its use, and herein, we have taken the more conservative approach of reporting the simplest kinetic model.

In their broadband transient absorption experiments, Karunakaran et al. reported an ultrafast lifetime of about $200 \mathrm{fs}$, which was assigned to movement of the wavepacket along the 
$\mathrm{L}_{\mathrm{a}}$ state potential energy surface from a planar region to a shallow minimum. ${ }^{24}$ From this minimum, the excited population reaches a conical intersection with the ground state, through ring puckering at the $\mathrm{C} 2$ atom, ${ }^{24}$ in agreement with recent calculations for GMP. ${ }^{28}$ This deformation of planarity allows for internal conversion back to the ground state, corresponding with the second lifetime of approximately $800 \mathrm{fs}$. Not surprisingly, the average of both lifetimes agrees well with our reported lifetime of $680 \pm 40 \mathrm{fs}$, suggesting this lifetime may simply be the weighted mean of the two exponential lifetimes reported by Karunakaran et al. Hence, the $680 \mathrm{fs}$ lifetime reported herein is directly associated with the $\tau_{1}$ and $\tau_{2}$ dynamics reported in this early work (i.e., wavepacket motion along the almost flat $\mathrm{L}_{\mathrm{a}}$ potential energy surface $\left(\tau_{1}\right)$ and internal conversion through the $\mathrm{L}_{\mathrm{a}} / \mathrm{S}_{0}$ conical intersection $\left(\tau_{2}\right)$ ). The third lifetime of ca. 2 ps, was assigned to vibrational cooling in the ground state, ${ }^{24}$ also in good agreement with our assignment.

\section{Comparison of the excited-state dynamics in $7 \mathrm{dza}$ and GMP}

As shown in Scheme 1, substitution of the nitrogen atom at the seven position of the guanine chromophore by a $\mathrm{C}-\mathrm{H}$ group gives rise to the $7 \mathrm{dza}$ nucleobase. One of the motivations of this study is to probe if this simple substitution affects the excited state dynamics observed in the guanine monomers, particularly in GMP. Hence, we performed steady-state, time-resolved, and computational calculations for both molecules under equal experimental and computational conditions. The following relaxation mechanism is proposed for the 7 dza nucleoside, $\mathrm{S}_{2}\left(\mathrm{~L}_{\mathrm{b}}\right) \rightarrow$ $\mathrm{S}_{1}\left(\mathrm{~L}_{\mathrm{a}}\right) \rightarrow \mathrm{S}_{0}$, whereas $\mathrm{S}_{2}\left(\mathrm{~L}_{\mathrm{b}}\right) \rightarrow \mathrm{S}_{1}\left(\mathrm{~L}_{\mathrm{a}}\right) \rightarrow \mathrm{S}_{0}($ hot $) \rightarrow \mathrm{S}_{0}$ is proposed for GMP. At first sight, both mechanisms appear to be qualitatively similar. However, the global and target analyses reported in this study demonstrate that there are quantitative differences between both molecules. 
For $7 \mathrm{dza}$, we propose that internal conversion from the $\mathrm{L}_{\mathrm{b}}$ to $\mathrm{L}_{\mathrm{a}}$ state occurs within ca. $210 \mathrm{fs}$, whereas we propose that this internal conversion pathway cannot be resolved for GMP with our experimental setup (i.e., it should occur in a $<100 \mathrm{fs}$ time scale). This suggest that there may be a small energy barrier to access the conical intersection between the $\mathrm{L}_{\mathrm{b}}$ and $\mathrm{L}_{\mathrm{a}}$ states in 7dza that is relatively larger than in GMP. Similarly, internal conversion from the $\mathrm{L}_{\mathrm{a}}$ state to the ground state occurs within ca. $680 \mathrm{fs}$ in GMP, populating the vibrational-excited ground state. However, internal conversion from the $\mathrm{L}_{\mathrm{a}}$ to the ground state occurs in ca. $1.4 \mathrm{ps}$ in $7 \mathrm{dza}$. Furthermore, we are unable to observe vibrational cooling dynamics in the ground state for this molecule, even though its steady-state absorption spectrum is red shifted compared to that of GMP (Figure 1). One unlikely possibility is that vibrational cooling dynamics is occurring in a similar time scale as internal conversion to the ground state, which would not allow us to resolve both processes in our broadband transient experiments. Performing other time-resolved experiments such as fluorescence up-conversion or femtosecond time-resolved IR could evaluate this possibility. According to our assignments, internal conversion from the $\mathrm{L}_{\mathrm{a}}$ to the ground state in $7 \mathrm{dza}$ occurs in about 2.6-fold slower time scale than in GMP. This also suggests that there is a relatively larger energy barrier to access the conical intersection between the $\mathrm{L}_{\mathrm{a}}$ state and the ground state in 7dza than in GMP. Comparisons of the excited state dynamics for both molecules at select probe traces are shown in Figure S7, supporting the idea that the overall relaxation dynamics are slower in 7dza than in GMP.

\section{Conclusions}

In this study, steady-state and time-resolved absorption experiments were combined with vertical excitation energies to reveal the excited-state dynamics of $7 \mathrm{dza}$ in aqueous solution upon excitation at $267 \mathrm{~nm}$. The results were compared directly with those obtained for GMP under 
equal experimental and computational conditions. It was shown that substitution of a single nitrogen for a methine $(\mathrm{C}-\mathrm{H})$ group at position seven of the guanine moiety stabilizes the ${ }^{1} \pi \pi^{*} \mathrm{~L}_{b}$ and $\mathrm{L}_{\mathrm{a}}$ states of $7 \mathrm{dza}$ and alters the topology of their potential energy surfaces in such a way that the population dynamics of both internal conversion pathways in $7 \mathrm{dza}$ are significantly slowed down compared to those in GMP. Multiconfigurational excited-state calculations and molecular dynamics simulations are necessary to provide further insights about the electronic relaxation mechanism in $7 \mathrm{dza}$.

\section{Experimental and Computational Methodology}

\section{A. Materials and steady-state spectroscopy}

Guanosine 5'-monophosphate disodium salt (GMP, 97\% purity) and 7-deazaguanosine (7dza, 98\% purity) were purchased from Acros and Biosynth Carbosynth, respectively, and were used as received. Phosphate buffer solutions with total phosphate concentration of $20 \mathrm{mM}$ from monosodium and disodium phosphate salts dissociated in ultrapure water (Millipore) were freshly prepared the day of each laser irradiation experiment. The $\mathrm{pH}$ of the solution was adjusted using $0.1 \mathrm{M}$ solutions of $\mathrm{NaOH}$ and $\mathrm{HCl}$ to the desired $\mathrm{pH}$ of 6.8 ( $\pm 0.1 \mathrm{pH}$ units). Steady-state absorption was recorded using a Cary 100 spectrometer.

\section{B. Quantum chemical calculations}

Quantum chemical calculations were performed using Gaussian 16 suite of programs. ${ }^{33}$ Ground state optimizations were performed at the B3LYP/IEFPCM/6-311+G(d,p) level of theory for both $7 \mathrm{dza}$ and GMP in water. For each molecule, the calculations were performed with the sugar in both the syn- and anti-conformations (see Tables S1 and S2). A sodium atom was added as a counterion in the calculations of GMP to neutralize the negative charge of the phosphate 
group. Vertical excitation energies were calculated at the TD-PBE0/IEFPCM/6-311+G(d,p) level of theory. A polarizable continuum model (PCM) using the integral equation formalism (IEF) was included in the ground state optimization and for the calculations of the vertical excitation energies to model the bulk solvent dielectric effect of water on each molecule. ${ }^{34,35}$

\section{Transient absorption spectroscopy}

The experimental setup and data analysis used for the femtosecond broadband transient absorption spectroscopy (TAS) technique have been described in great deal elsewhere. ${ }^{36-38}$ Briefly, the TAS spectrometer (Helios, Ultrafast Systems) make use of a Ti:Sapphire oscillator (Vitesse, Coherent), which seeds a regenerative amplifier (Libra-HE, Coherent) producing $100 \mathrm{fs}$ pulses, centered at $800 \mathrm{~nm}$, and with a $1 \mathrm{kHz}$ repetition rate. Generation of the $267 \mathrm{~nm}$ excitation pulse was done as described previously, ${ }^{7}$ by pumping an optical rail kit (FKE series, EKSMA optics). A translating $2 \mathrm{~mm} \mathrm{CaF}_{2}$ window was used to generate the white light continuum in the spectral probe window from 320 to $700 \mathrm{~nm}$.

The absorbance of the sample solutions at the excitation wavelength was matched at 1.3 for both GMP and 7dza during TAS experiments. The homogeneity of the solutions in a $2 \mathrm{~mm}$ path length fused silica cell was maintained by continuous stirring with a Teflon-coated magnetic stirbar. To minimize the putative contamination of the transient signals by the formation of any photoproducts absorbing at $267 \mathrm{~nm}$, freshly prepared samples were used, and frequently refreshed over the course of data acquisition such that the absorption of the samples at $267 \mathrm{~nm}$ did not change by more than 3 percent.

Data collection made use of a home-made LabView program, while global and target data analyses were performed using the Glotaran graphical user interface to the R-package TIMP 
software. ${ }^{39}$ The full multidimensional data set for both GMP and 7dza was globally fit using a two compartment sequential kinetic model convoluted with a Gaussian instrument response function of $250 \pm 50 \mathrm{fs}(\mathrm{FWHM})$. The evolution associated difference spectra (EADS) were extracted from the global and target analysis. ${ }^{39}$

\section{Experimental pump-probe conditions used to eliminate the two-photon hydrated electron signal from the buffer solvent}

In order to eliminate the need to correct for the two-photon ionization of water upon excitation of the sample solutions at $267 \mathrm{~nm},{ }^{32}$ the photon densities were adjusted to ensure no parasitic signal from the characteristic solvated electron ${ }^{40}$ were observed. To accomplish this, the probe beam size was kept constant at ca. 100 microns $(0.110 \mathrm{~mm} \pm 0.005)$, while the pump beam size was increased to ca. $1.2 \mathrm{~mm}(1.160 \mathrm{~mm} \pm 0.005)$ using the knife edge method to obtain the $1 / \mathrm{e}^{2}$ beam diameter ${ }^{41}$ Briefly, a razor blade and micrometer translation stage is used to cover $15 \%$ and $85 \%$ of the beam power while noting the micrometer position, where the difference between the two measurements yields the desired beam diameter. The reported value is an average of a series of three measurements. Once the pump beam size was widened to the desired beam size, the average pump intensity was increased to $2 \mu \mathrm{J}$ at the cell to improve signal to noise of the recorded data. An average photon density or quantum flux of $2.5 \times 10^{17}$ photons $/ \mathrm{s} \cdot \mathrm{cm}^{2}$ was estimated and is further detailed in the SI. The fraction of photons absorbed by 7dza or GMP was maximized by using an absorbance of 1.3 at $267 \mathrm{~nm}$, while simultaneously eliminating the formation of solvated electron signal. This can be observed in Figure 5, where the neat buffer solvent and adenosine 5'-monophosphate (AMP) were used to verify that no hydrated electron signal was observed under the experimental conditions used in this work. 
We note that the broadband data for AMP can be globally fitted using a monoexponential kinetic model (Figure 6; see also Figure S8), yielding a lifetime of $(0.39 \pm 0.02)$ ps. Interestingly, this value compares favorably with the lifetime reported by Kohler and co-workers in two independent studies for AMP in aqueous buffer solution of $(0.30 \pm 0.02) \mathrm{ps}^{32}$ and $(0.33 \pm$ 0.03) $\mathrm{ps}^{42}$. However, in their broadband transient absorption experiments exciting at $267 \mathrm{~nm}$, Kwok et al. ${ }^{43}$ reported two lifetimes of 0.13 and 0.45 ps for adenosine in aqueous solution, whereas Pecourt et al. ${ }^{44}$ reported a single lifetime of $(0.29 \pm 0.04)$ ps for adenosine in the same solvent. It is conceivable that the mono-exponential decay lifetime reported in this and other ${ }^{32,42}$ studies for AMP upon excitation at $267 \mathrm{~nm}$ is simply the weighted mean of the two exponential lifetimes determined Kwok et al. ${ }^{43}$ Alternatively, the one-photon signal from the water solvent upon excitation at $267 \mathrm{~nm}$ may contribute to the additional lifetime reported by these authors.
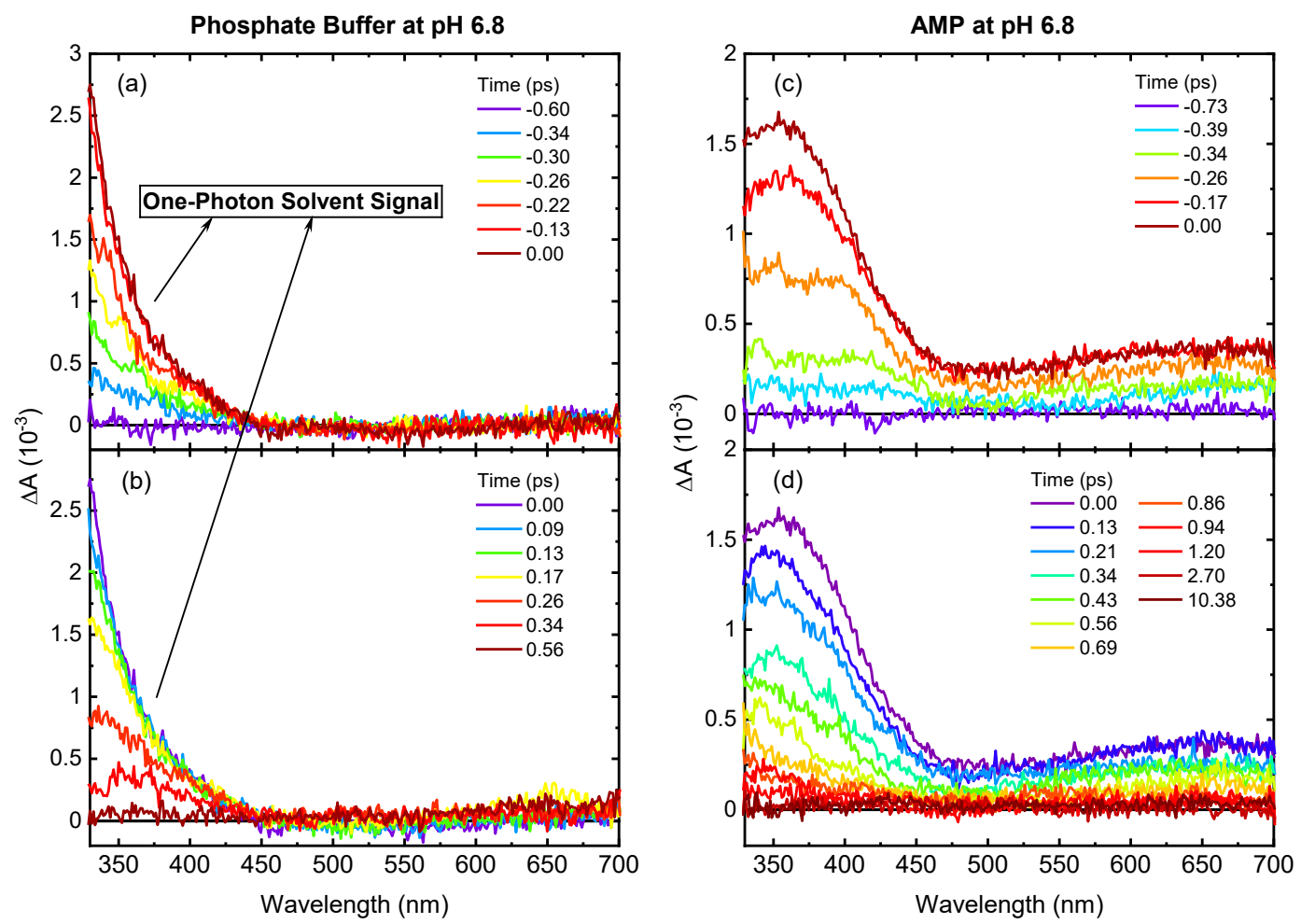

Figure 5. Spectral evolution of the transient absorption spectra of neat buffer (left) and adenosine 5'-monophosphate (AMP, right) in phosphate buffer $\mathrm{pH} 6.8$ following excitation at $267 \mathrm{~nm}$. 

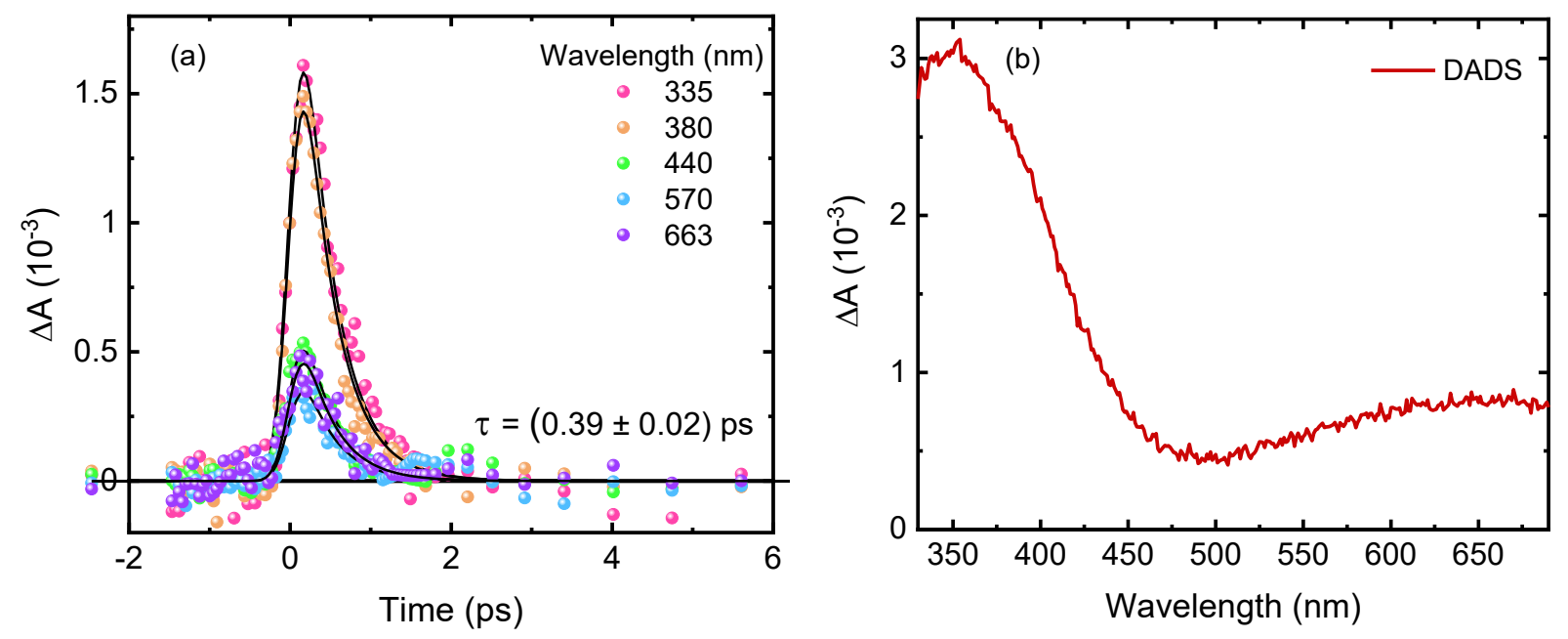

Figure 6. (a) Representative kinetic decay traces of AMP at select probe wavelengths globally fitted with a mono-exponential kinetic model. (b) Decay associated difference spectra (DADS) of AMP globally fitted with a mono-exponential kinetic model.

As shown in Figure 5, the experimental conditions used in this work eliminate the detection of the two-photon ionization of water in the phosphate buffer, but a solvent signal is still observed in the spectral range from 320 to $450 \mathrm{~nm}$ (Figure 5a,b). As shown in Figure 7, the amplitude of this signal increases linearly with the laser pump power. 


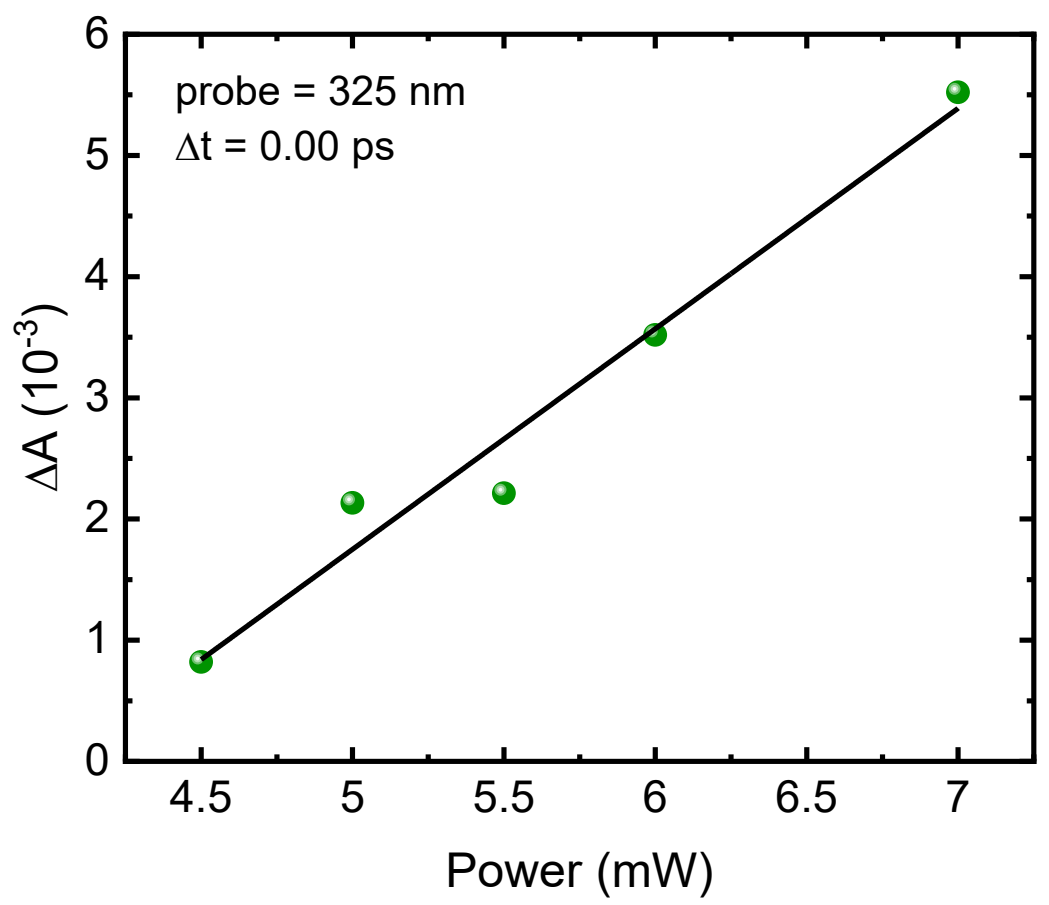

Figure 7. Dependence of the phosphate buffer signal at the probe wavelength of $325 \mathrm{~nm}$ with the average laser pump power at $267 \mathrm{~nm}$.

To determine whether a correction for the one-photon signal of the solvent was necessary under the experimental conditions used, an investigation was carried out by scaling the onephoton signal of the buffer-only to $5 \%$ (i.e., equivalent to the fraction of photons absorbed by the solvent in the GMP and 7dza solutions), and then subtracting the one-photon solvent signal from the transient absorption data of $7 \mathrm{dza}$ and GMP. This correction was observed to result in a negligible change in the transient absorption spectra of both 7dza and GMP (i.e., decreasing the $\Delta \mathrm{A}$ amplitude of the transient absorption spectra by less than $1 \%$ in the spectral probe region below $450 \mathrm{~nm}$ and significantly less in the spectral region from 450 to $700 \mathrm{~nm}$ ). 


\section{Supplementary Material}

DFT-optimized geometries and TD-DFT vertical excitation energies and Kohn-Sham orbitals for each conformation of 7dza and GMP; estimation of the population reaching the $\mathrm{L}_{\mathrm{a}}$ and $\mathrm{L}_{\mathrm{b}}$ states upon excitation of 7dza and GMP at $267 \mathrm{~nm}$; normalized decay traces at different probe wavelengths; equations and procedures used to estimate the photon flux at $267 \mathrm{~nm}$.

\section{Dedication}

This paper is dedicated to Distinguished University Professor Mary D. Barkley, a superb biophysical chemist who developed and applied innovative approaches to investigate protein and DNA structure and interactions. She is an influential mentor and advocate for women and underrepresented groups in the sciences. Barkley was first woman professor in the Department of Chemistry and the first woman to hold an endowed chair in the natural sciences at CWRU.

\section{Acknowledgements}

The authors acknowledge the National Science Foundation (Grant No. CHE-1800052). This work made use of the High-Performance Computing Resource in the Core Facility for Advanced Research Computing at CWRU.

\section{Data Availability}

The data that support the findings of this study are available from the corresponding author upon reasonable request.

\section{References}

${ }^{1}$ C.E. Crespo-Hernández, B. Cohen, P.M. Hare, and B. Kohler, Chem. Rev. 104, 1977 (2004). 
${ }^{2}$ C.T. Middleton, K. de La Harpe, C. Su, Y.K. Law, C.E. Crespo-Hernández, and B. Kohler, Annu. Rev. Phys. Chem. 60, 217 (2009).

${ }^{3}$ A.A. Beckstead, Y. Zhang, M.S. De Vries, and B. Kohler, Phys. Chem. Chem. Phys. 18, 24228 (2016).

${ }^{4}$ C. Reichardt, C. Wen, R.A. Vogt, and C.E. Crespo-Hernández, Photochem. Photobiol. Sci. 12, $1341(2013)$.

${ }^{5}$ C.E. Crespo-Hernández, L. Martínez-Fernández, C. Rauer, C. Reichardt, S. Mai, M. Pollum, P. Marquetand, L. González, and I. Corral, J. Am. Chem. Soc. 137, 4368 (2015).

${ }^{6}$ L. Martínez-Fernández, S. Arslancan, D. Ivashchenko, C.E. Crespo-Hernández, and I. Corral, Phys. Chem. Chem. Phys. 21, 13467 (2019).

${ }^{7}$ E.M. Arpa, M.M. Brister, S.J. Hoehn, C.E. Crespo-Hernández, and I. Corral, J. Phys. Chem. Lett. 11, 5156 (2020).

${ }^{8}$ S. Yamazaki, A.L. Sobolewski, and W. Domcke, Phys. Chem. Chem. Phys. 11, 10165 (2009).

${ }^{9}$ B. Cohen, P.M. Hare, and B. Kohler, J. Am. Chem. Soc. 125, 13594 (2003).

${ }^{10}$ K. Röttger, R. Siewertsen, and F. Temps, Chem. Phys. Lett. 536, 140 (2012).

${ }^{11}$ J.P. Villabona-Monsalve, R. Noria, S. Matsika, and J. Peón, J. Am. Chem. Soc. 134, 7820 (2012).

${ }^{12}$ M. Barbatti, A.J.A. Aquino, J.J. Szymczak, D. Nachtigallová, P. Hobza, and H. Lischka, Proc. Natl. Acad. Sci. U. S. A. 107, 21453 (2010).

13 J. Chen and B. Kohler, Phys. Chem. Chem. Phys. 14, 10677 (2012). 
${ }^{14}$ S. Matsika, Photoinduced Phenomena in Nucleic Acids I (Springer International Publishing, 2015).

${ }^{15}$ C.E. Pollum, Marvin; Martinez-Fernandez, Lara; Crespo-Hernandez, Photoinduced

Phenomena in Nucleic Acids I (Springer International Publishing, 2015).

${ }^{16}$ F.D. Lewis, Photochem. Photobiol. 81, 65 (2005).

${ }^{17}$ N. Gorczak, T. Fujii, A.K. Mishra, A.J. Houtepen, F.C. Grozema, and F.D. Lewis, J. Phys. Chem. B 119, 7673 (2015).

${ }^{18}$ F.D. Lewis, P. Daublain, B. Cohen, J. Vura-Weis, and M.R. Wasielewski, Angew. Chemie Int. Ed. 47, 3798 (2008).

${ }^{19}$ F.D. Lewis, R.M. Young, and M.R. Wasielewski, Acc. Chem. Res. 51, 1746 (2018).

${ }^{20}$ S.O. Kelley and J.K. Barton, Chem. Biol. 5, 413 (1998).

${ }^{21}$ A.K. Thazhathveetil, M.A. Harris, R.M. Young, M.R. Wasielewski, and F.D. Lewis, J. Am. Chem. Soc. 139, 1730 (2017).

${ }^{22}$ F.D. Lewis, J. Liu, X. Liu, X. Zuo, R.T. Hayes, and M.R. Wasielewski, Angew. Chemie - Int. Ed. 41, 1026 (2002).

${ }^{23}$ F.D. Lewis, X. Liu, J. Liu, S.E. Miller, R.T. Hayes, and M.R. Wasielewski, Nature 406, 51 (2000).

${ }^{24}$ V. Karunakaran, K. Kleinermanns, R. Improta, and S.A. Kovalenko, J. Am. Chem. Soc. 131, 5839 (2009).

${ }^{25}$ C.C.-W. Cheng, C. Ma, C.T.-L. Chan, K.Y.-F. Ho, and W.-M. Kwok, Photochem. Photobiol. 
Sci. 12, 1351 (2013).

${ }^{26}$ F.A. Miannay, T. Gustavsson, A. Banyasz, and D. Markovitsi, J. Phys. Chem. A 114, 3256 (2010).

${ }^{27}$ J. Lee, J.R. Challa, and D.W. McCamant, J. Phys. Chem. B 121, 4722 (2017).

${ }^{28}$ S.F. Altavilla, J. Segarra-Mart Ã-, A. Nenov, I. Conti, I. Rivalta, and M. Garavelli, Front. Chem. 3, 1 (2015).

${ }^{29}$ L. Serrano-Andrés, M. Merchán, and A.C. Borin, J. Am. Chem. Soc. 130, 2473 (2008).

${ }^{30}$ R. Krishnamurthy, Acc. Chem. Res. 45, 2035 (2012).

${ }^{31}$ J.R. Platt, J. Chem. Phys. 17, 484 (1949).

${ }^{32}$ C.E. Crespo-Hernández and B. Kohler, J. Phys. Chem. B 108, 11182 (2004).

${ }^{33}$ M.J. Frisch, G.W. Trucks, H.B. Schlegel, G.E. Scuseria, M. a. Robb, J.R. Cheeseman, G. Scalmani, V. Barone, G. a. Petersson, H. Nakatsuji, X. Li, M. Caricato, a. V. Marenich, J. Bloino, B.G. Janesko, R. Gomperts, B. Mennucci, H.P. Hratchian, J. V. Ortiz, a. F. Izmaylov, J.L. Sonnenberg, Williams, F. Ding, F. Lipparini, F. Egidi, J. Goings, B. Peng, A. Petrone, T. Henderson, D. Ranasinghe, V.G. Zakrzewski, J. Gao, N. Rega, G. Zheng, W. Liang, M. Hada, M. Ehara, K. Toyota, R. Fukuda, J. Hasegawa, M. Ishida, T. Nakajima, Y. Honda, O. Kitao, H. Nakai, T. Vreven, K. Throssell, J. a. Montgomery Jr., J.E. Peralta, F. Ogliaro, M.J. Bearpark, J.J. Heyd, E.N. Brothers, K.N. Kudin, V.N. Staroverov, T. a. Keith, R. Kobayashi, J. Normand, K. Raghavachari, a. P. Rendell, J.C. Burant, S.S. Iyengar, J. Tomasi, M. Cossi, J.M. Millam, M. Klene, C. Adamo, R. Cammi, J.W. Ochterski, R.L. Martin, K. Morokuma, O. Farkas, J.B. Foresman, and D.J. Fox, (2016). 
${ }^{34}$ E. Cancès, B. Mennucci, and J. Tomasi, J. Chem. Phys. 107, 3032 (1997).

${ }^{35}$ V. Barone, M. Cossi, and J. Tomasi, J. Chem. Phys. 107, 3210 (1997).

${ }^{36}$ C. Reichardt, R.A. Vogt, and C.E. Crespo-Hernández, J. Chem. Phys. 131, 0 (2009).

${ }^{37}$ M. Pollum, S. Jockusch, and C.E. Crespo-Hernández, J. Am. Chem. Soc. 136, 17930 (2014).

${ }^{38}$ M.M. Brister and C.E. Crespo-Hernández, J. Phys. Chem. Lett. 10, 2156 (2019).

${ }^{39}$ J.J. Snellenburg, S. Laptenok, R. Seger, K.M. Mullen, and I.H.M. van Stokkum, J. Stat. Softw. 49, 1 (2012).

${ }^{40}$ P.M. Hare, E.A. Price, and D.M. Bartels, J. Phys. Chem. A 112, 6800 (2008).

${ }^{41}$ Y. Suzaki and A. Tachibana, Appl. Opt. 14, 2809 (1975).

${ }^{42}$ C.E. Crespo-Hernández, B. Cohen, and B. Kohler, Nature 436, 1141 (2005).

${ }^{43}$ W.-M. Kwok, C. Ma, and D.L. Phillips, J. Am. Chem. Soc. 128, 11894 (2006).

44 J.M.L. Pecourt, J. Peon, and B. Kohler, J. Am. Chem. Soc. 123, 10370 (2001). 


\section{Excited State Dynamics of 7-Deazaguanosine and Guanosine 5'-Monophosphate in Aqueous Solution}

Sarah E. Krul, ${ }^{1, \dagger}$ Sean J. Hoehn, ${ }^{1, \dagger}$ Karl Feierabend,,${ }^{1,2}$ Carlos E. Crespo-Hernández ${ }^{*}, 1$

${ }^{1}$ Department of Chemistry, Case Western Reserve University, Cleveland, Ohio 44106, USA

${ }^{2}$ Permanent address: Department of Chemistry, The College of Wooster, Wooster, Ohio 44691, USA

* email of corresponding author: carlos.crespo@case.edu, ${ }^{\dagger}$ these authors contributed equally to this work

\section{Supplementary Results}

Table S1. Optimized geometries of 7dza conformations calculated at the TD-PBE0/IEFPCM/6$311+\mathrm{G}(\mathrm{d}, \mathrm{p}) / / \mathrm{B} 3 \mathrm{LYP} / \mathrm{IEFPCM} / 6-311+\mathrm{G}(\mathrm{d}, \mathrm{p})$ level of theory and zero point energy corrected.

(5'syn)-7dza

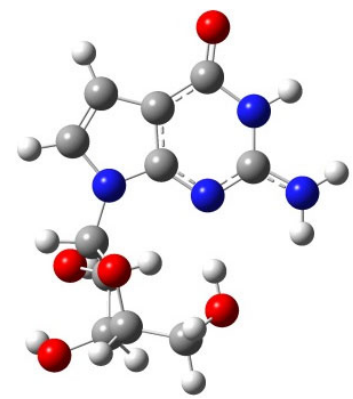

$0 \mathrm{kcal} / \mathrm{mol}$ (2’syn)-7dza

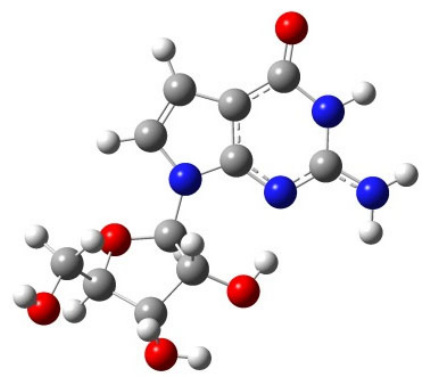

$2.7 \mathrm{kcal} / \mathrm{mol}$ (anti)-7dza

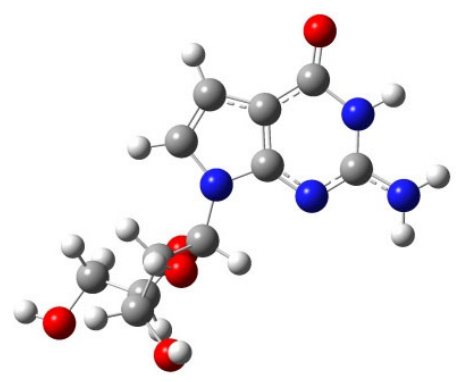


Table S2. Optimized geometries of GMP conformations using sodium as a counter ion for the negatively charged phosphate group calculated at the TD-PBE0/IEFPCM/6-

$311+\mathrm{G}(\mathrm{d}, \mathrm{p}) / / \mathrm{B} 3 \mathrm{LYP} / \mathrm{IEFPCM} / 6-311+\mathrm{G}(\mathrm{d}, \mathrm{p})$ level of theory and zero point energy corrected.

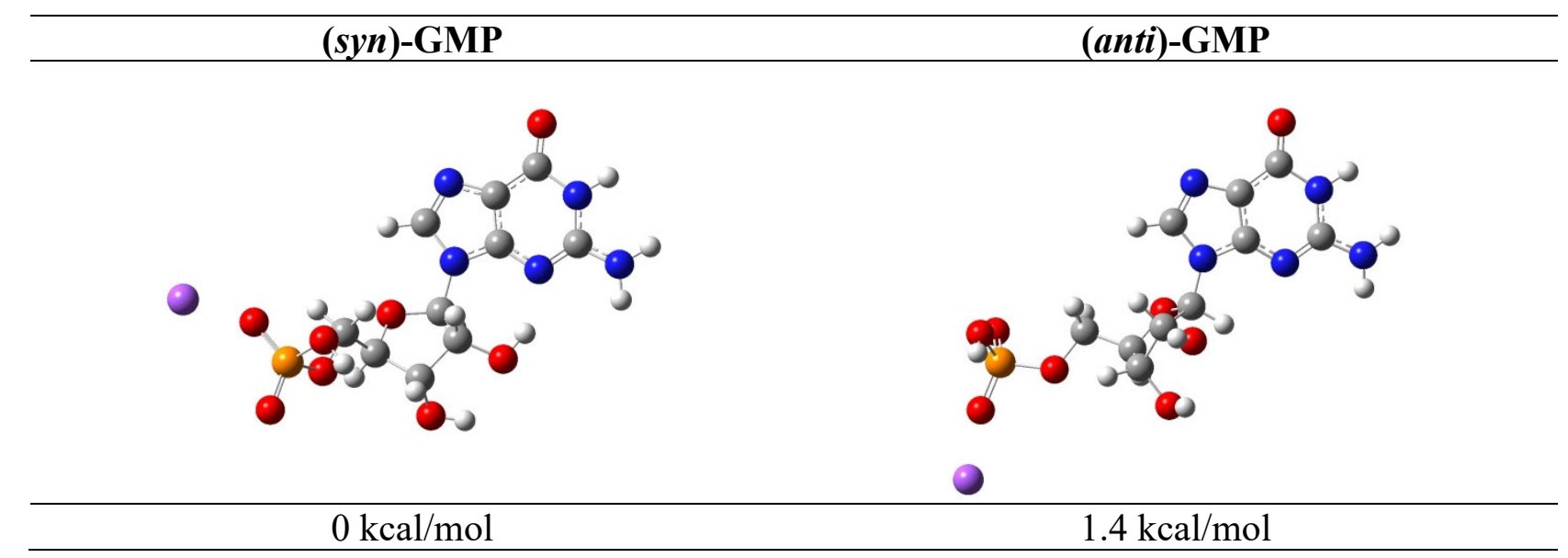


Table S3. Vertical energies for the relevant singlet and triplet transitions of 5'syn-7dza with the 5 '-OH of the ribose oriented toward the N1 purine position calculated at the TD-

PBE0/IEFPCM/6-311+G(d,p)//B3LYP/IEFPCM/6-311+G(d,p) level of theory and zero point energy corrected. Primary character of the transition showed in parentheses.

\begin{tabular}{|l|l|l|l|}
\hline State & Transitions & $\begin{array}{l}\text { \% } \\
\text { Contribution }\end{array}$ & Energy (eV) \\
\hline $\mathbf{S}_{\mathbf{1}}\left(\boldsymbol{\pi} \boldsymbol{\pi}^{*}\right)$ & $\mathrm{H}-0 \rightarrow \mathrm{L}+0$ & 97.1 & $4.66(0.1448)$ \\
& $\mathrm{H}-0 \rightarrow \mathrm{L}+1$ & 2.9 & \\
\hline $\mathbf{S}_{\mathbf{2}}\left(\boldsymbol{\pi} \boldsymbol{\pi}^{*}\right)$ & $\mathrm{H}-1 \rightarrow \mathrm{L}+0$ & 4.2 & $4.98(0.3044)$ \\
& $\mathrm{H}-0 \rightarrow \mathrm{L}+0$ & 2.8 & \\
& $\mathrm{H}-0 \rightarrow \mathrm{L}+1$ & 93.0 & 3.55 \\
\hline $\mathbf{T}_{\mathbf{1}}\left(\boldsymbol{\pi} \boldsymbol{\pi}^{*}\right)$ & $\mathrm{H}-0 \rightarrow \mathrm{L}+0$ & 46.6 & \\
& $\mathrm{H}-0 \rightarrow \mathrm{L}+1$ & 53.4 & 3.61 \\
\hline $\mathbf{T}_{\mathbf{2}}\left(\boldsymbol{\pi} \boldsymbol{\pi}^{*}\right)$ & $\mathrm{H}-0 \rightarrow \mathrm{L}+0$ & 56.9 & 4.62 \\
& $\mathrm{H}-0 \rightarrow \mathrm{L}+1$ & 43.1 & \\
\hline $\mathbf{T}_{\mathbf{3}}\left(\boldsymbol{\pi} \boldsymbol{\pi}^{*}\right)$ & $\mathrm{H}-8 \rightarrow \mathrm{L}+1$ & 8.4 & \\
& $\mathrm{H}-6 \rightarrow \mathrm{L}+0$ & 3.4 & \\
& $\mathrm{H}-1 \rightarrow \mathrm{L}+0$ & 6.8 & \\
& $\mathrm{H}-1 \rightarrow \mathrm{L}+1$ & 6.5 & \\
& $\mathrm{H}-0 \rightarrow \mathrm{L}+1$ & 5.3 & \\
& $\mathrm{H}-0 \rightarrow \mathrm{L}+6$ & 9.2 & \\
& $\mathrm{H}-0 \rightarrow \mathrm{L}+7$ & 44.7 & \\
& $\mathrm{H}-0 \rightarrow \mathrm{L}+8$ & 10.3 & \\
& $\mathrm{H}-0 \rightarrow \mathrm{L}+11$ & 5.4 & \\
\hline
\end{tabular}




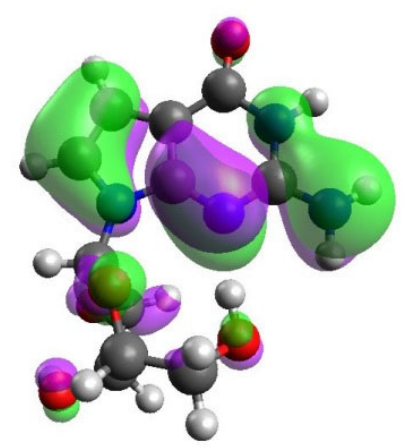

H-8

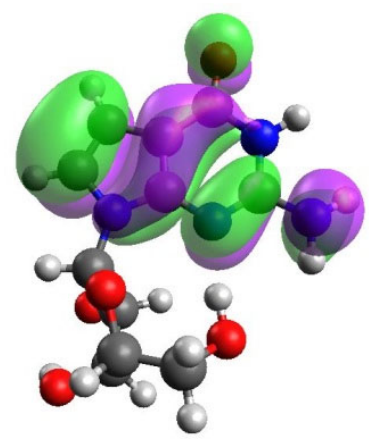

H-O

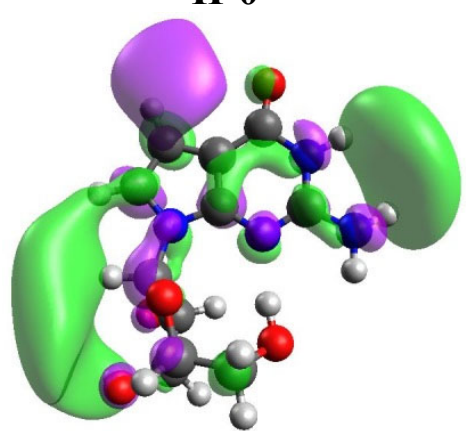

$\mathrm{L}+6$

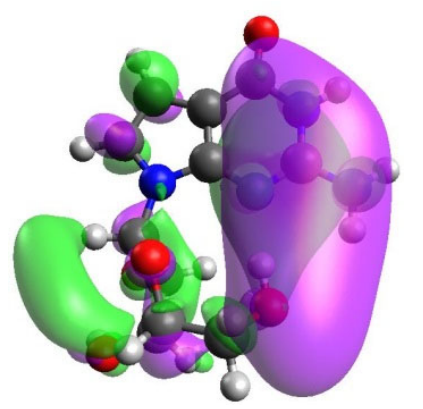

$\mathbf{L}+11$

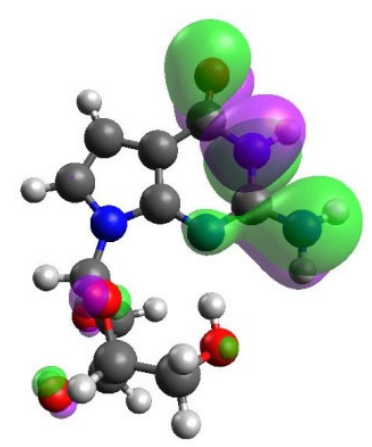

H-6

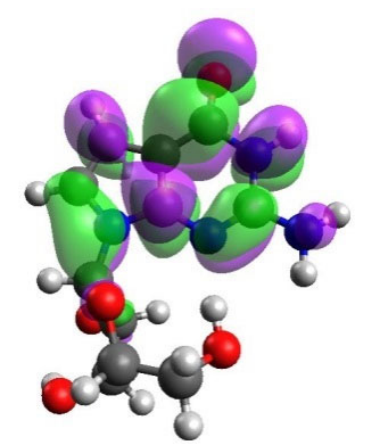

L+0

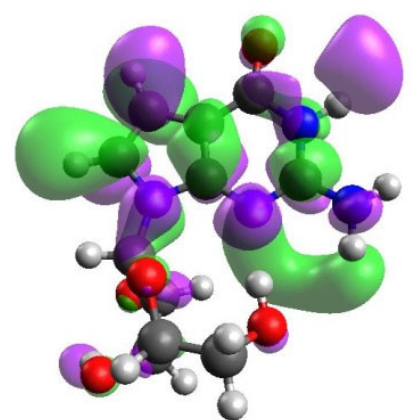

L+7

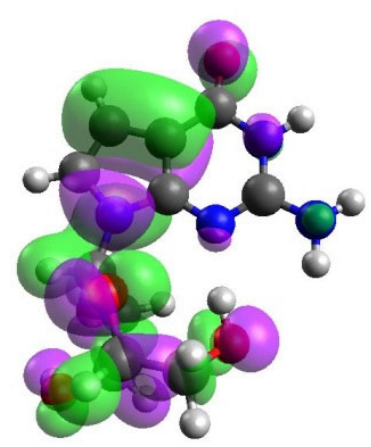

H-1

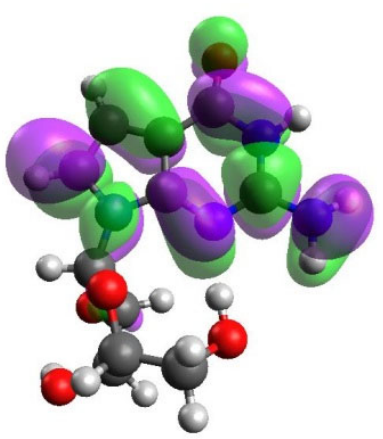

$\mathbf{L}+1$

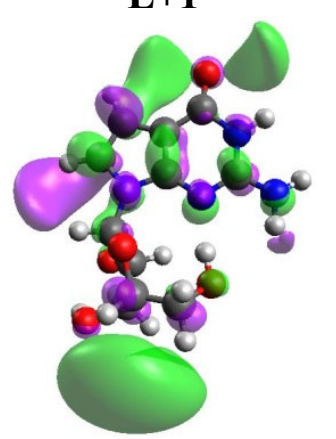

$\mathbf{L}+8$

Figure S1. Kohn-Sham orbitals that contribute to the relevant singlet and triplet vertical transitions of syn-7dza with the 5' $-\mathrm{OH}$ of the ribose oriented toward the $\mathrm{N} 1$ purine position calculated at the TD-PBE0/IEFPCM/6-311+G(d,p)//B3LYP/IEFPCM/6-311+G(d,p) level of theory and zero point energy corrected. 
Table S4. Vertical energies for the relevant singlet and triplet transitions of syn-GMP calculated at the TD-PBE0/IEFPCM/6-311+G(d,p)//B3LYP/IEFPCM/6-311+G(d,p) level of theory and zero point energy corrected. Primary character of the transition showed in parentheses.

\begin{tabular}{|l|l|l|l|}
\hline State & Transitions & $\begin{array}{l}\text { \% } \\
\text { Contribution }\end{array}$ & Energy (eV) \\
\hline $\mathbf{S}_{\mathbf{1}}\left(\boldsymbol{\pi} \boldsymbol{\pi}^{*}\right)$ & $\mathrm{H}-0 \rightarrow \mathrm{L}+0$ & 85.8 & $4.90(0.1541)$ \\
& $\mathrm{H}-0 \rightarrow \mathrm{L}+1$ & 14.2 & \\
\hline $\mathbf{S}_{\mathbf{2}}\left(\boldsymbol{\pi} \boldsymbol{\pi}^{*}\right)$ & $\mathrm{H}-0 \rightarrow \mathrm{L}+0$ & 14.2 & $5.18(0.3668)$ \\
& $\mathrm{H}-0 \rightarrow \mathrm{L}+1$ & 85.8 & \\
\hline $\mathbf{T}_{\mathbf{1}}\left(\boldsymbol{\pi} \boldsymbol{\pi}^{*}\right)$ & $\mathrm{H}-0 \rightarrow \mathrm{L}+0$ & 97.8 & 3.63 \\
& $\mathrm{H}-0 \rightarrow \mathrm{L}+1$ & 2.2 & \\
\hline $\mathbf{T}_{\mathbf{2}}\left(\boldsymbol{\pi} \boldsymbol{\pi}^{*}\right)$ & $\mathrm{H}-0 \rightarrow \mathrm{L}+0$ & 2.8 & 3.91 \\
& $\mathrm{H}-0 \rightarrow \mathrm{L}+1$ & 97.2 & 4.82 \\
\hline $\mathbf{T}_{\mathbf{3}}\left(\boldsymbol{\pi} \boldsymbol{\pi}^{*}\right)$ & $\mathrm{H}-14 \rightarrow \mathrm{L}+0$ & 4.4 & \\
& $\mathrm{H}-14 \rightarrow \mathrm{L}+1$ & 3.9 & \\
& $\mathrm{H}-9 \rightarrow \mathrm{L}+0$ & 2.9 & \\
& $\mathrm{H}-0 \rightarrow \mathrm{L}+5$ & 3.2 & \\
& $\mathrm{H}-0 \rightarrow \mathrm{L}+7$ & 26.2 & \\
& $\mathrm{H}-0 \rightarrow \mathrm{L}+8$ & 25.4 & \\
& $\mathrm{H}-0 \rightarrow \mathrm{L}+9$ & 7.7 & \\
& $\mathrm{H}-0 \rightarrow \mathrm{L}+10$ & 23.5 & \\
& $\mathrm{H}-0 \rightarrow \mathrm{L}+13$ & 2.9 & \\
\hline
\end{tabular}




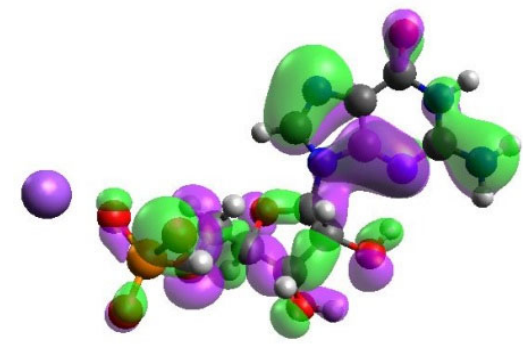

H-14
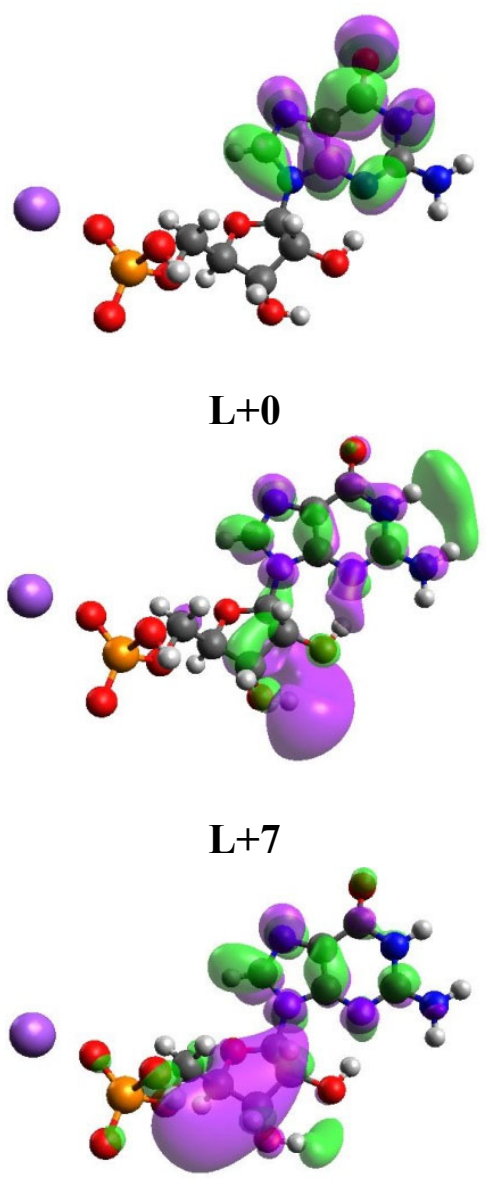

$\mathbf{L}+\mathbf{1 0}$

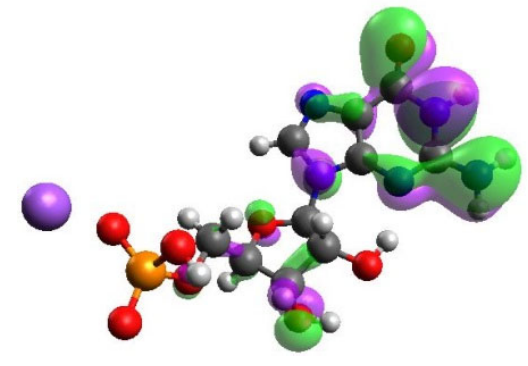

H-9

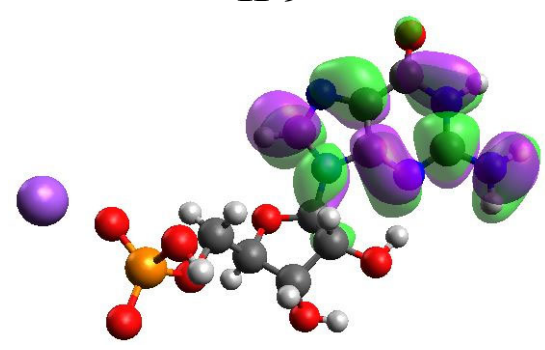

L+1

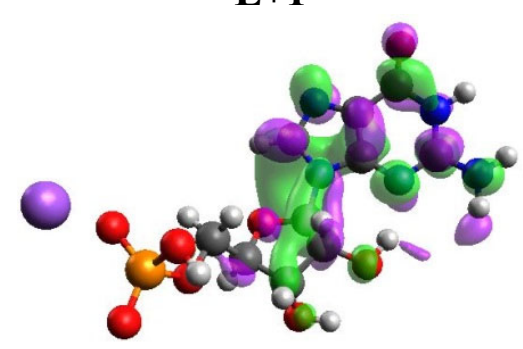

$\mathbf{L}+\mathbf{8}$

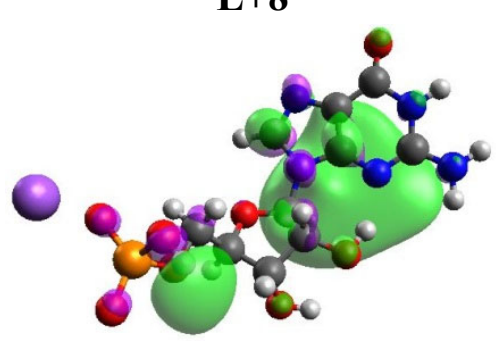

$\mathbf{L}+\mathbf{1 3}$

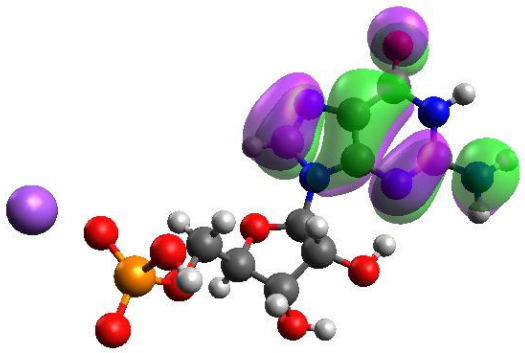

H-0

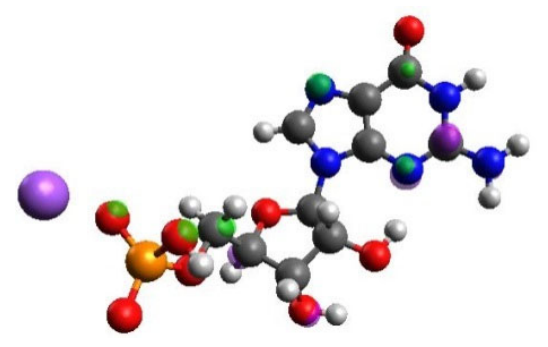

$\mathbf{L}+5$

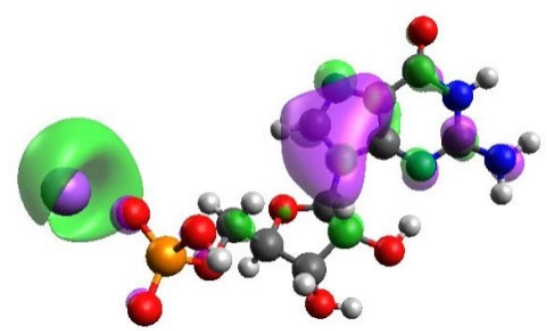

$\mathbf{L}+9$

Figure S2. Kohn-Sham orbitals that contribute to the relevant singlet and triplet vertical transitions of syn-GMP calculated at the TD-PBE0/IEFPCM/6-311+G(d,p)//B3LYP/IEFPCM/6$311+\mathrm{G}(\mathrm{d}, \mathrm{p})$ level of theory and zero point energy corrected. 
Table S5. Vertical energies for the relevant singlet and triplet transitions of anti-7dza calculated at the TD-PBE0/IEFPCM/6-311+G(d,p)//B3LYP/IEFPCM/6-311+G(d,p) level of theory and zero point energy corrected. Primary character of the transition showed in parentheses.

\begin{tabular}{|l|l|l|l|}
\hline State & Transitions & $\begin{array}{l}\text { \% } \\
\text { Contribution }\end{array}$ & Energy (eV) \\
\hline $\mathbf{S}_{\mathbf{1}}\left(\boldsymbol{\pi} \boldsymbol{\pi}^{*}\right)$ & $\mathrm{H}-0 \rightarrow \mathrm{L}+0$ & 85.8 & $4.71(0.1554)$ \\
& $\mathrm{H}-0 \rightarrow \mathrm{L}+1$ & 14.2 & \\
\hline $\mathbf{S}_{\mathbf{2}}\left(\boldsymbol{\pi} \boldsymbol{\pi}^{*}\right)$ & $\mathrm{H}-0 \rightarrow \mathrm{L}+0$ & 14.2 & $5.0(0.3556)$ \\
& $\mathrm{H}-0 \rightarrow \mathrm{L}+1$ & 85.8 & \\
\hline $\mathbf{T}_{\mathbf{1}}\left(\boldsymbol{\pi} \boldsymbol{\pi}^{*}\right)$ & $\mathrm{H}-0 \rightarrow \mathrm{L}+0$ & 13.3 & 3.52 \\
& $\mathrm{H}-0 \rightarrow \mathrm{L}+1$ & 86.7 & \\
\hline $\mathbf{T}_{\mathbf{2}}\left(\boldsymbol{\pi} \boldsymbol{\pi}^{*}\right)$ & $\mathrm{H}-0 \rightarrow \mathrm{L}+0$ & 87.6 & 3.66 \\
& $\mathrm{H}-0 \rightarrow \mathrm{L}+1$ & 12.4 & \\
\hline $\mathbf{T}_{\mathbf{3}}\left(\boldsymbol{\pi} \boldsymbol{\pi}^{*}\right)$ & $\mathrm{H}-8 \rightarrow \mathrm{L}+1$ & 5.1 & \\
& $\mathrm{H}-7 \rightarrow \mathrm{L}+1$ & 2.8 & \\
& $\mathrm{H}-6 \rightarrow \mathrm{L}+0$ & 2.7 & \\
& $\mathrm{H}-3 \rightarrow \mathrm{L}+1$ & 4.0 & \\
& $\mathrm{H}-1 \rightarrow \mathrm{L}+0$ & 4.4 & \\
& $\mathrm{H}-1 \rightarrow \mathrm{L}+1$ & 4.7 & \\
& $\mathrm{H}-0 \rightarrow \mathrm{L}+1$ & 5.1 & \\
& $\mathrm{H}-0 \rightarrow \mathrm{L}+6$ & 18.7 & \\
& $\mathrm{H}-0 \rightarrow \mathrm{L}+7$ & 27.1 & \\
& $\mathrm{H}-0 \rightarrow \mathrm{L}+8$ & 14.4 & \\
& $\mathrm{H}-0 \rightarrow \mathrm{L}+9$ & 10.9 & \\
\hline
\end{tabular}




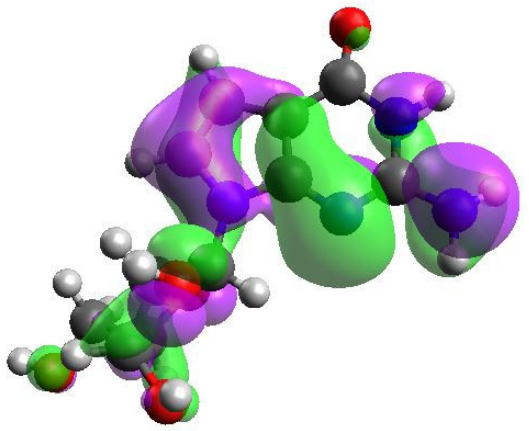

H-8

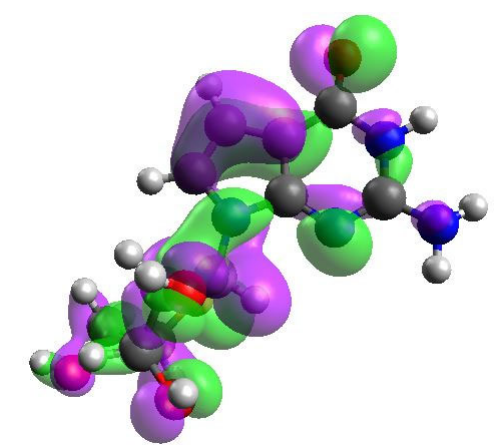

H-3

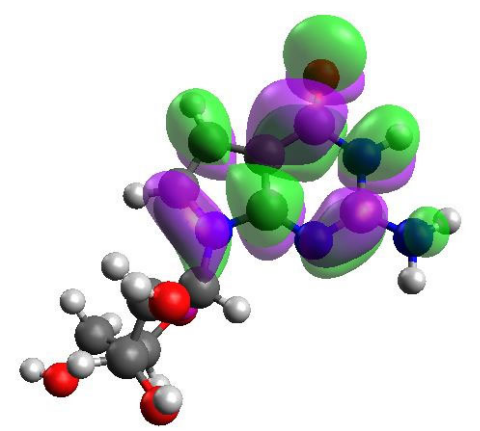

L+0

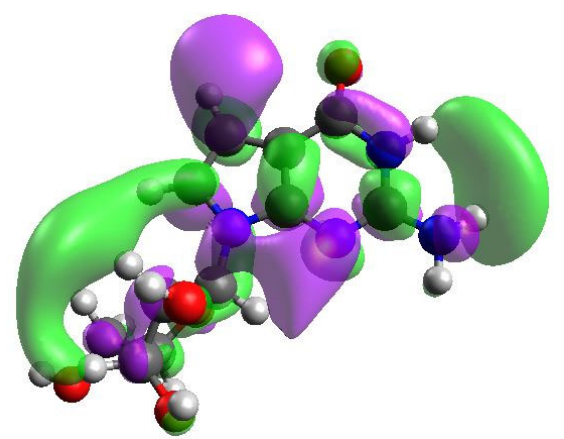

L+7

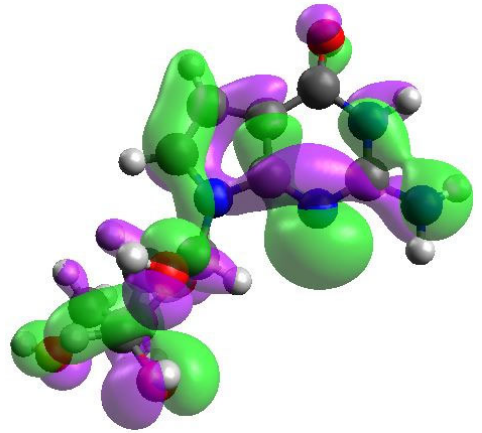

H-7

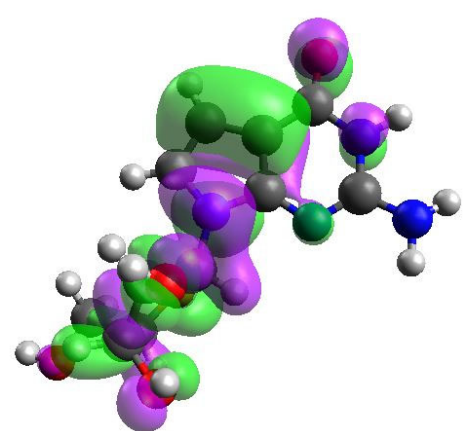

H-1

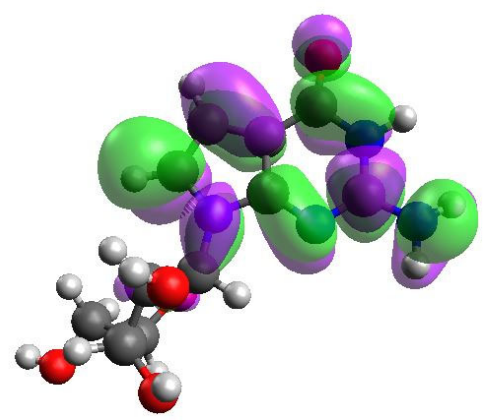

L+1

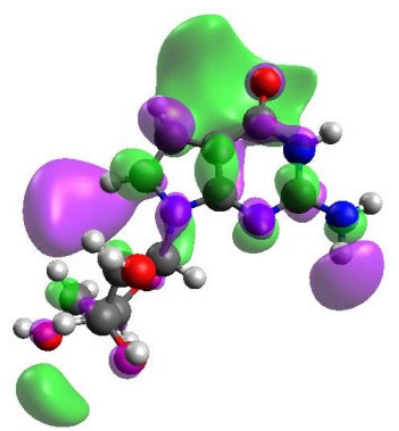

L+8

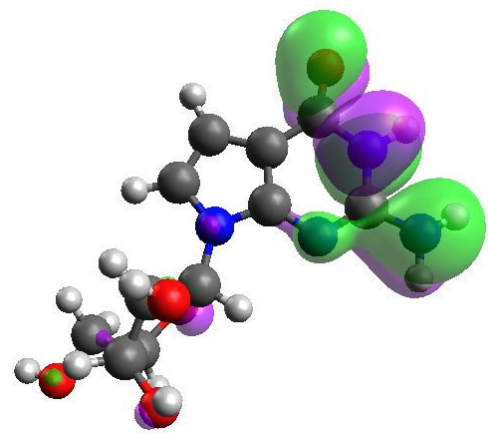

H-6

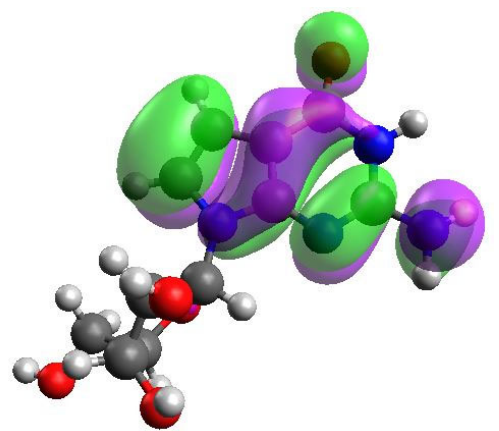

H-0

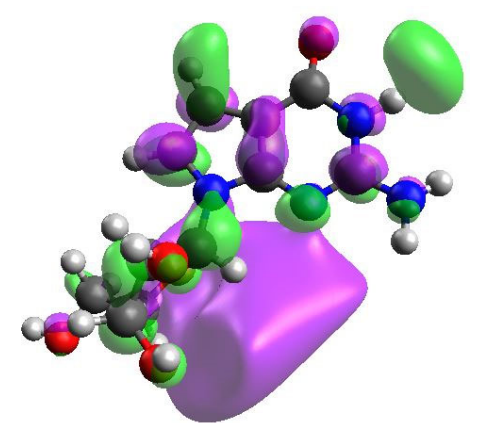

L+6

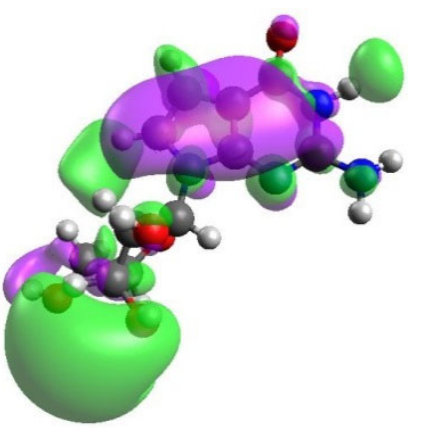

L+9

Figure S3. Kohn-Sham orbitals that contribute to the relevant singlet and triplet vertical transitions of anti-7dza calculated at the TD-PBE0/IEFPCM/6-311+G(d,p)//B3LYP/IEFPCM/6$311+\mathrm{G}(\mathrm{d}, \mathrm{p})$ level of theory and zero point energy corrected. 
Table S6. Vertical energies for the relevant singlet and triplet transitions of anti-GMP calculated at the TD-PBE0/IEFPCM/6-311+G(d,p)//B3LYP/IEFPCM/6-311+G(d,p) level of theory and zero point energy corrected. Primary character of the transition showed in parentheses.

\begin{tabular}{|l|l|l|l|}
\hline State & Transitions & $\begin{array}{l}\text { \% } \\
\text { Contribution }\end{array}$ & Energy (eV) \\
\hline $\mathbf{S}_{\mathbf{1}}\left(\boldsymbol{\pi} \boldsymbol{\pi}^{*}\right)$ & $\mathrm{H}-0 \rightarrow \mathrm{L}+0$ & 79.2 & $4.90(0.156)$ \\
& $\mathrm{H}-0 \rightarrow \mathrm{L}+1$ & 20.8 & \\
\hline $\mathbf{S}_{\mathbf{2}}\left(\boldsymbol{\pi} \boldsymbol{\pi}^{*}\right)$ & $\mathrm{H}-0 \rightarrow \mathrm{L}+0$ & 20.6 & $5.14(0.3959)$ \\
& $\mathrm{H}-0 \rightarrow \mathrm{L}+1$ & 79.4 & 3.63 \\
\hline $\mathbf{T}_{\mathbf{1}}\left(\boldsymbol{\pi} \boldsymbol{\pi}^{*}\right)$ & $\mathrm{H}-0 \rightarrow \mathrm{L}+0$ & 93.1 & \\
& $\mathrm{H}-0 \rightarrow \mathrm{L}+1$ & 6.9 & 3.85 \\
\hline $\mathbf{T}_{\mathbf{2}}\left(\boldsymbol{\pi} \boldsymbol{\pi}^{*}\right)$ & $\mathrm{H}-0 \rightarrow \mathrm{L}+0$ & 8.1 & \\
& $\mathrm{H}-0 \rightarrow \mathrm{L}+1$ & 91.9 & 4.71 \\
$\mathbf{T}_{\mathbf{3}}\left(\boldsymbol{\pi} \boldsymbol{\pi}^{*}\right)$ & $\mathrm{H}-13 \rightarrow \mathrm{L}+0$ & 5.6 & \\
& $\mathrm{H}-13 \rightarrow \mathrm{L}+1$ & 5.2 & \\
& $\mathrm{H}-8 \rightarrow \mathrm{L}+0$ & 3.6 & \\
& $\mathrm{H}-0 \rightarrow \mathrm{L}+7$ & 28.9 & \\
& $\mathrm{H}-0 \rightarrow \mathrm{L}+8$ & 37.1 & \\
& $\mathrm{H}-0 \rightarrow \mathrm{L}+9$ & 16.7 & \\
& $\mathrm{H}-0 \rightarrow \mathrm{L}+10$ & 2.8 & \\
\hline
\end{tabular}




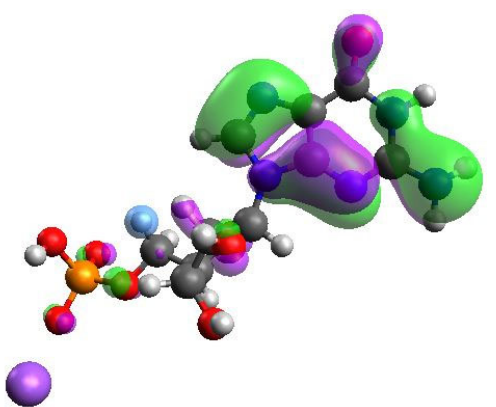

H-13

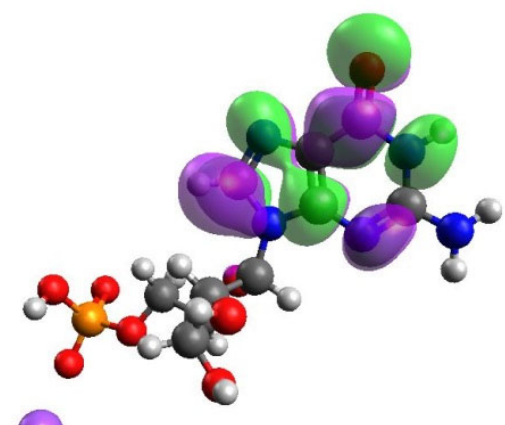

$\mathbf{L}+\mathbf{0}$

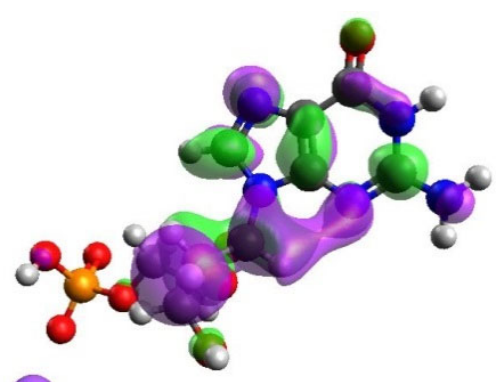

C

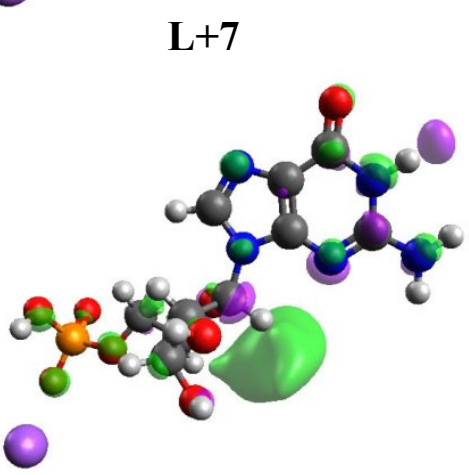

L+10

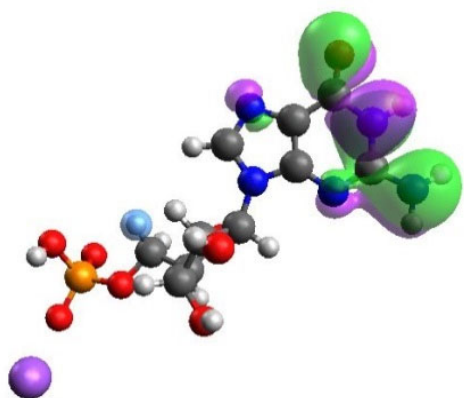

H-8

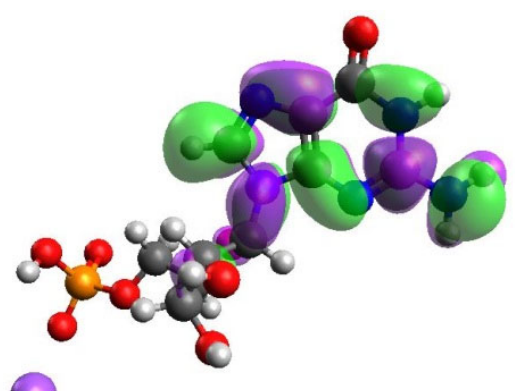

L+1

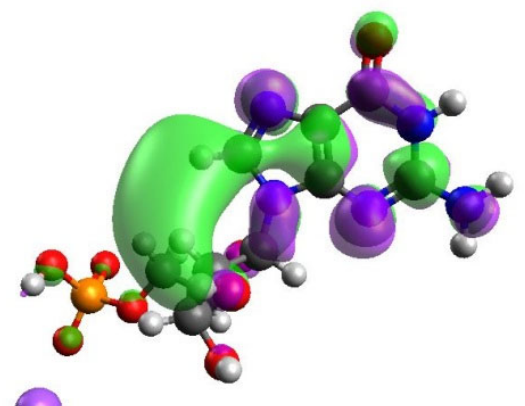

L+8

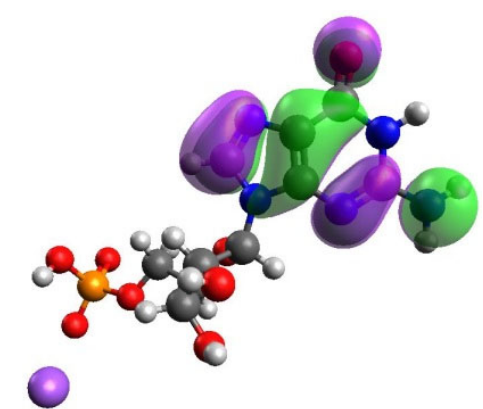

H-0

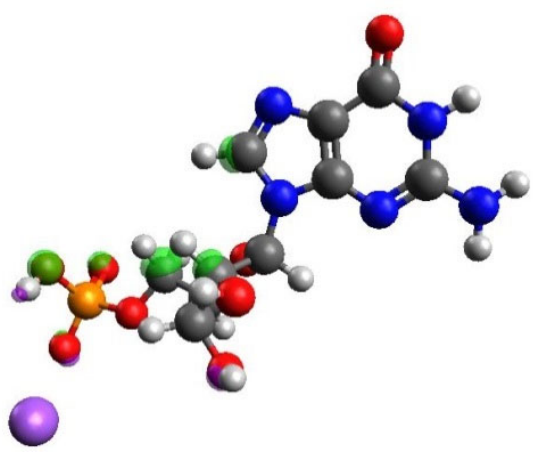

$\mathbf{L}+\mathbf{5}$

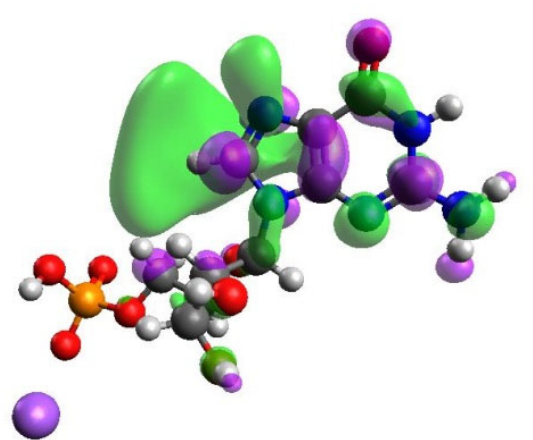

L+9

Figure S4. Kohn-Sham orbitals that contribute to the relevant singlet and triplet vertical transitions of syn-GMP calculated at the TD-PBE0/IEFPCM/6-311+G(d,p)//B3LYP/IEFPCM/6$311+\mathrm{G}(\mathrm{d}, \mathrm{p})$ level of theory and zero point energy corrected. 


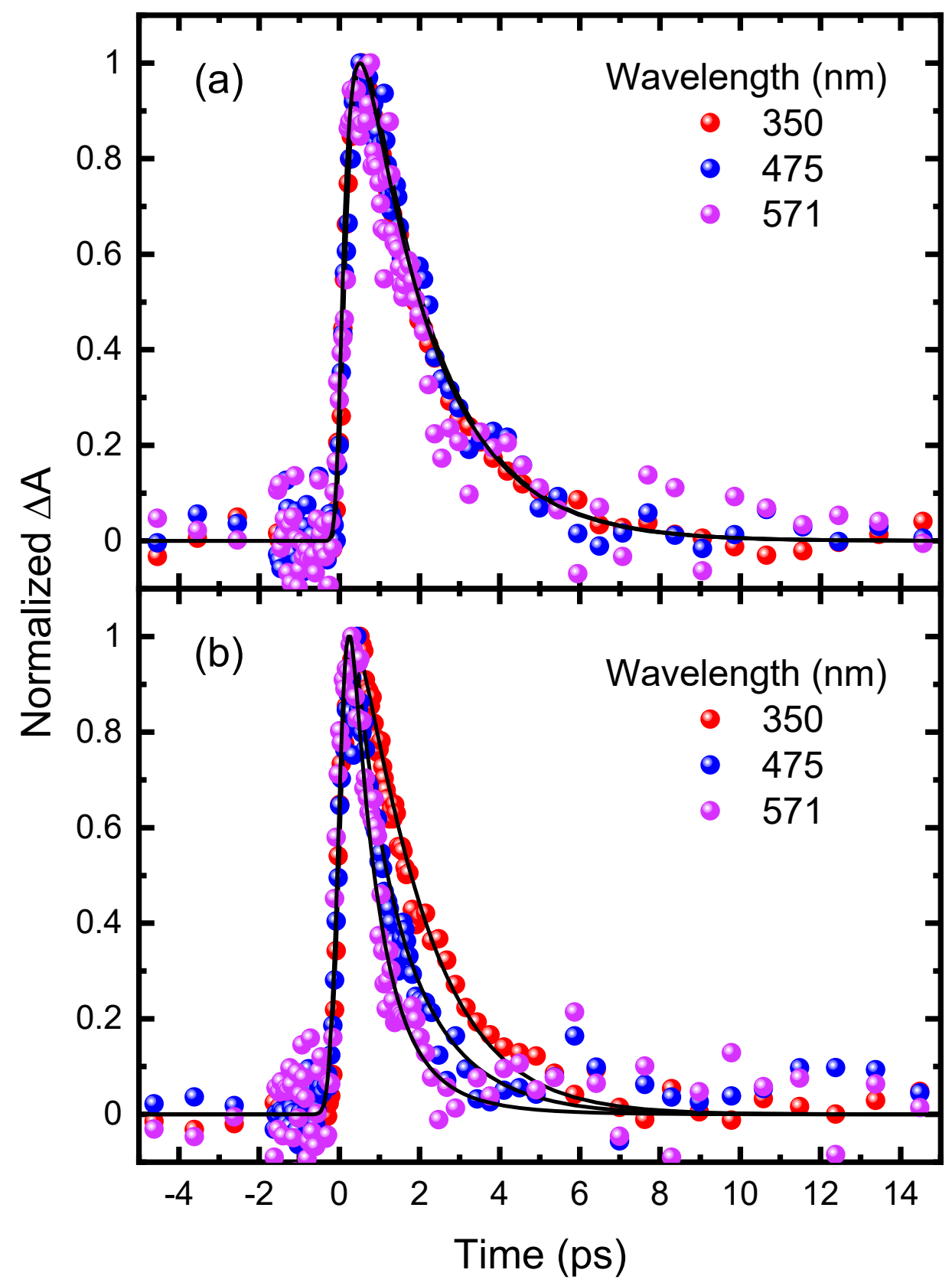

Figure S5. Normalized decay traces of (a) 7dza and (b) GMP at 350, 475, and $571 \mathrm{~nm}$ probe wavelengths. 


\section{Determination of state population following excitation at $267 \mathrm{~nm}$}

The lowest energy band of the steady-state absorption spectra for 7dza and GMP were deconvoluted to fit both the $\mathrm{L}_{a}$ and $\mathrm{L}_{b}$ states using a linear combination of two Gaussians functions. Using equation 1, the percent fraction of population that reach each respective excited singlet state upon excitation at $267 \mathrm{~nm}$ was estimated, where $\mathrm{X}$ and $\mathrm{Y}$ represent the respective $\mathrm{L}_{\mathrm{a}}$ and $\mathrm{Lb}$ states.

$\%$ fraction of population for state $X=\frac{\varepsilon_{X}(267 \mathrm{~nm})}{\varepsilon_{X}(267 \mathrm{~nm})+\varepsilon_{Y}(267 \mathrm{~nm})} \times 100$

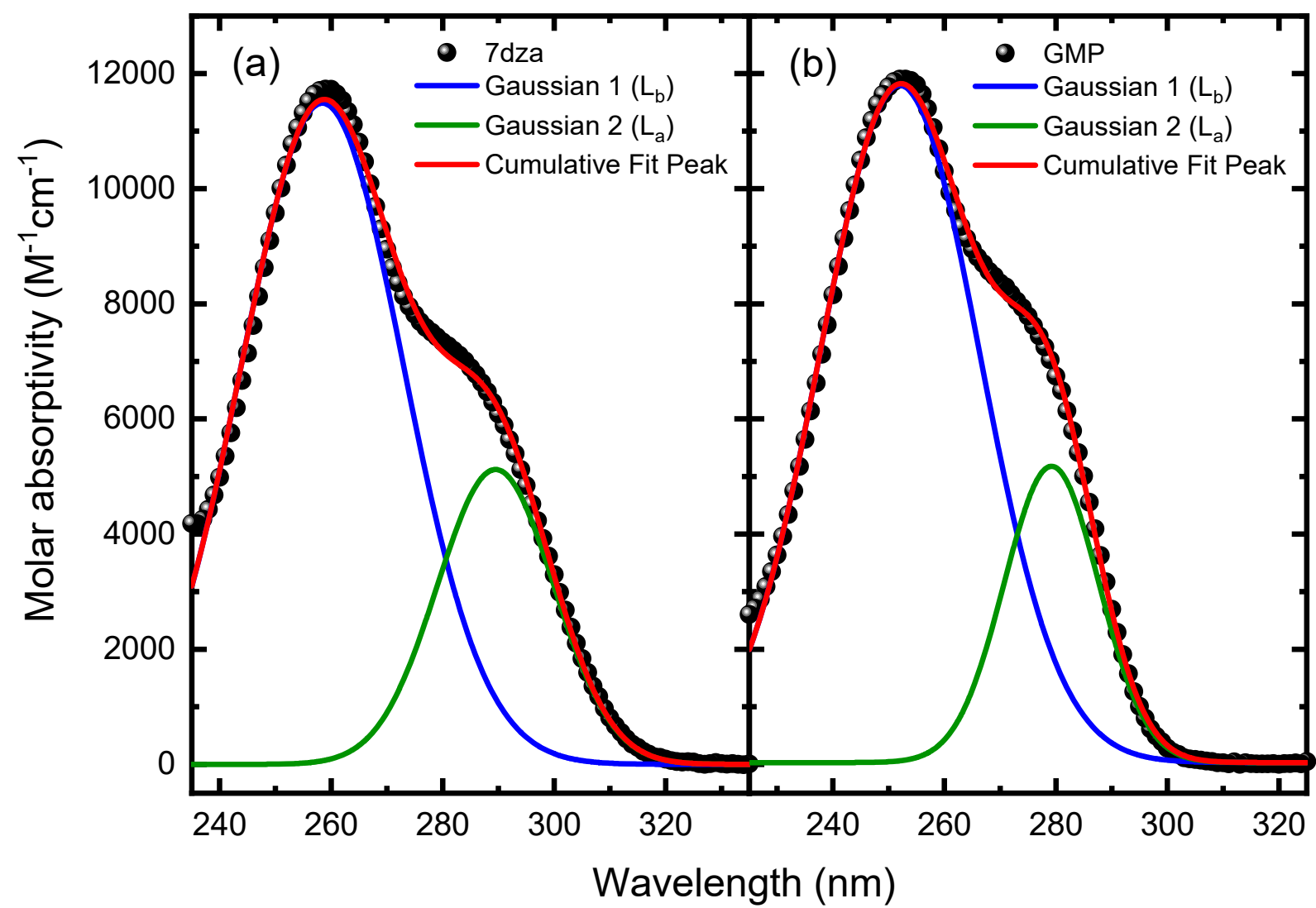

Figure S6. Deconvolution of the lowest energy absorption and into the $\mathrm{Lb}$ and $\mathrm{La}$ state for $7 \mathrm{dza}$ (left) and GMP (right) using a linear combination of two Gaussian functions. 

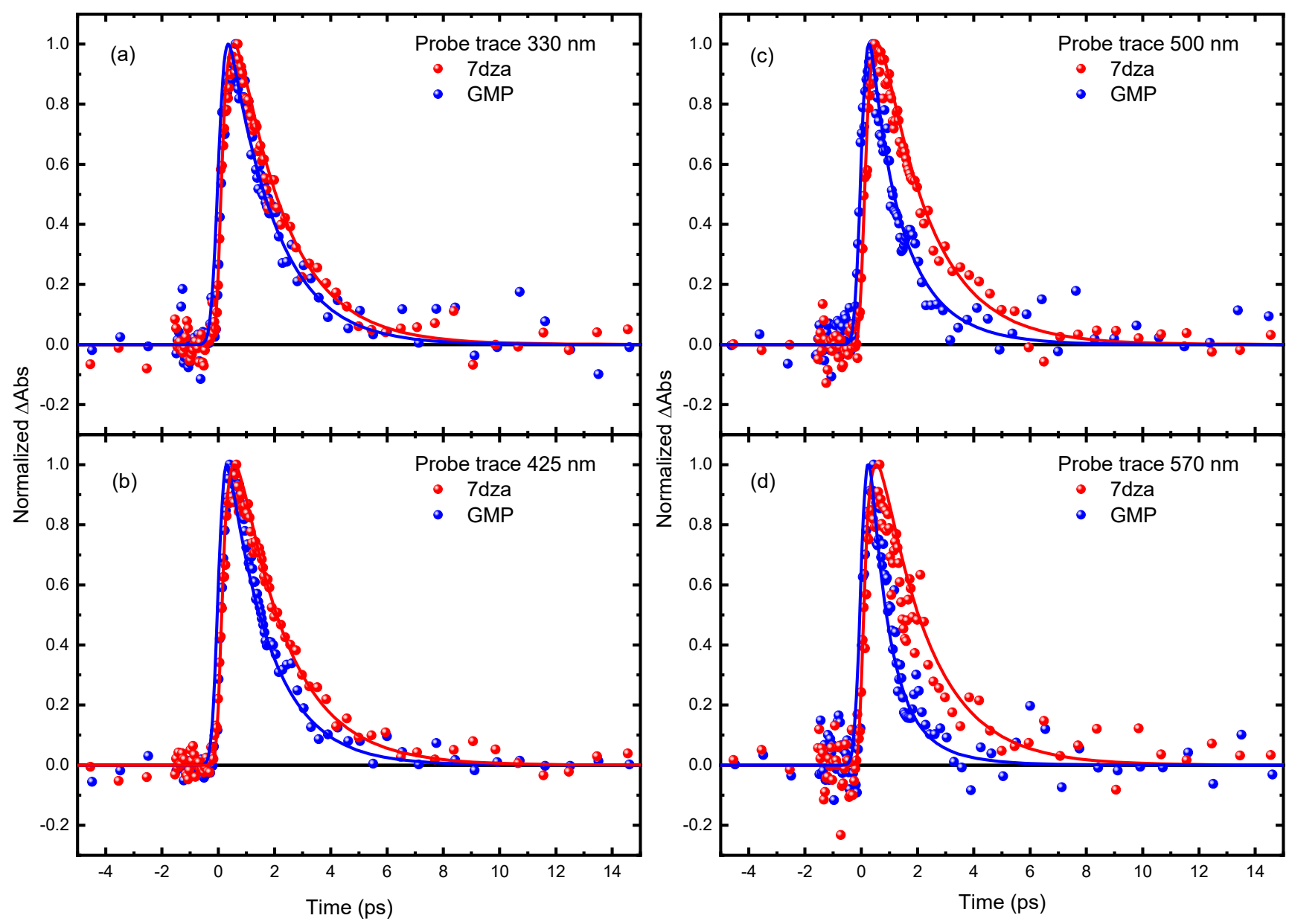

Figure S7. Normalized decay traces of 7dza and GMP at select probe wavelengths: a) $330 \mathrm{~nm}$, b) $425 \mathrm{~nm}$, c) $500 \mathrm{~nm}$, d) $570 \mathrm{~nm}$. 
Equations used for the calculation of the peak quantum flux at $267 \mathrm{~nm}$.

$$
\begin{aligned}
& \text { Energy }_{\text {per pulse }}(J)=\frac{\text { Power }_{\text {average }}(W)}{\text { Repetition }_{\text {rate }}(\mathrm{Hz})} \\
& \text { Peak }_{\text {Power }}(W)=\frac{\text { Energy }_{\text {perpulse }}(J)}{\text { Duration }_{\text {pulse }}(s)} \\
& \text { Intensity }\left(W / \mathrm{cm}^{2}\right)=\frac{\text { Peak }_{\text {Power }}(W)}{\text { Beam area }\left(\mathrm{cm}^{2}\right)}=\frac{\text { Peak }_{\text {Power }}}{\pi \cdot\left[\frac{\left(1 / e^{2} \text { diameter }\right)}{2}\right]^{2}} \cdot 2 \\
& \operatorname{Energy}_{\text {photon }}(J)=\frac{h \cdot c}{\lambda} \\
& \text { Quantum flux }\left(\text { photons } / \mathrm{cm}^{2} \cdot s\right)=\frac{\operatorname{Intensity~}\left(\mathrm{W} / \mathrm{cm}^{2}\right)}{\text { Energy }_{\text {photon }}(J)}
\end{aligned}
$$

The average power used in equation S2 was obtained using a silicon photodiode (S120VC ThorLabs). A value of $2.5 \times 10^{17}$ photons $/ \mathrm{s} \cdot \mathrm{cm}^{2}$ was estimated using a pump beam size of $1.2 \mathrm{~mm}$. An average estimated pulse duration of 275 femtoseconds (fs) was used for equations $\mathrm{S} 3$. This is due to the broadening of the $100 \mathrm{fs}$ pulse of the fundamental at $800 \mathrm{~nm}$ during the harmonic generation. Note the factor of 2 at the terminal end of equation S4. This multiplication factor is required because the peak power in the center of the beam is twice the average power density for an assumed Gaussian shaped beam. A peak photon density or quantum flux of $1.8 \times$ $10^{27}$ photons $/ \mathrm{s} \cdot \mathrm{cm}^{2}$ for the described conditions was thus obtained. 


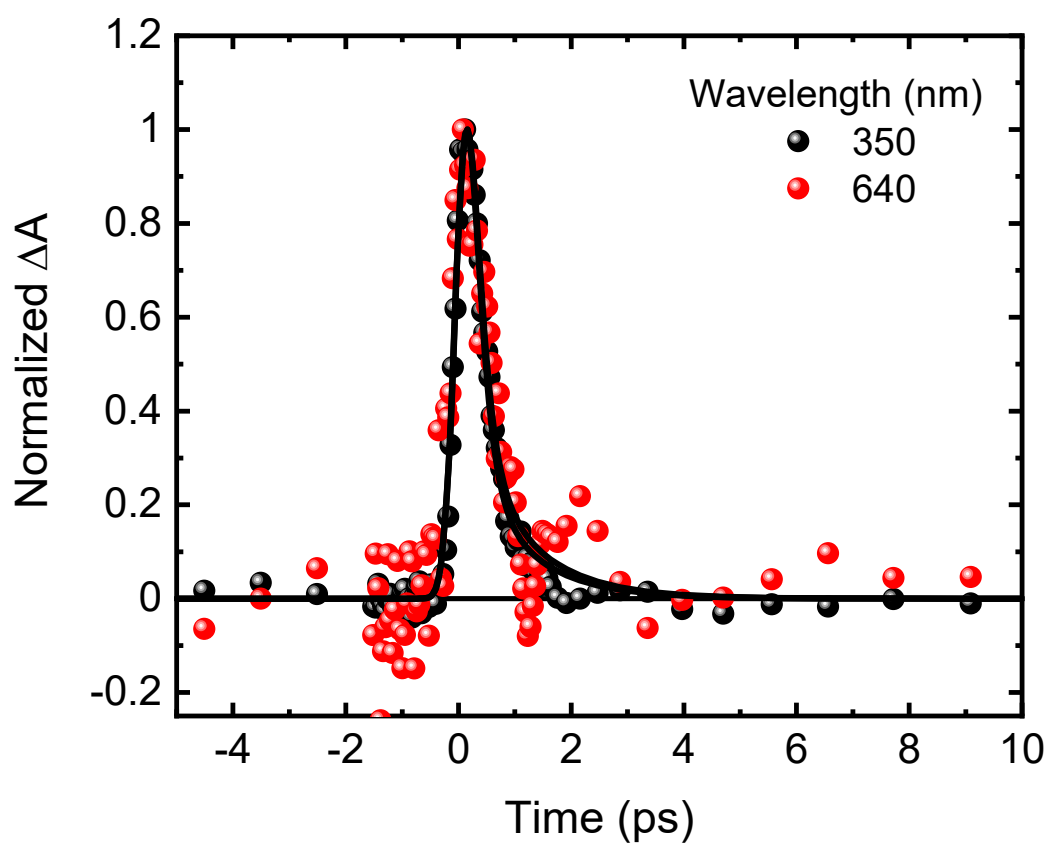

Figure S8. Normalized kinetic traces of AMP at 350 and $640 \mathrm{~nm}$ globally fit with a monoexponential kinetic model. 